\title{
Weak lensing analysis in three dimensions
}

\author{
P. G. Castro*, A. F. Heavens ${ }^{\dagger}$ and T. D. Kitching ${ }^{\ddagger}$ \\ Institute for Astronomy, University of Edinburgh, \\ Royal Observatory, Blackford Hill, Edinburgh EH9 3HJ, U.K.
}

(Dated: February 2, 2008)

\begin{abstract}
We present a comprehensive full-sky 3-dimensional analysis of the weak-lensing fields and their corresponding power spectra. Using the formalism of spin-weight spherical harmonics and spherical Bessel functions, we relate the two-point statistics of the harmonic expansion coefficients of the weak lensing shear and convergence to the power spectrum of the matter density perturbations, and derive small-angle limits. Such a study is relevant in view of the next generation of large-scale weak lensing surveys which will provide distance information about the sources through photometric redshifts. This opens up the possibility of accurate cosmological parameter estimation via weak lensing, with an emphasis on the equation of state of dark energy.

PACS numbers:
\end{abstract}

\section{INTRODUCTION}

Like the Cosmic Microwave Background (CMB) a decade ago, the study of gravitational weak lensing on a cosmic scale is now entering a promised golden age. As with the CMB, it is well understood theoretically [1, 2], it has now been detected (see e.g. $\underline{3},[4,[5,6,6],[]$ ) and ambitious experimental projects are under way. The uniqueness and thus the appeal of weak lensing lies in its clean and direct sensitivity to the total mass distribution of the Universe. Indeed, it arises from the deflection of light by the gravitational potential of the matter along the photon path, regardless of the precise nature or state of the intervening matter. With weak lensing one can hope to measure for the first time the 3D matter power spectrum over a wide range of scales, in principle independently of any model for evolution. It thus provides complementary information to that rendered by the CMB alone or other lower redshift cosmological probes. The CMB does provide the cleanest possible window into the physics of the early Universe and its parameters [9, 10], but lacks strong sensitivity to the Universe's subsequent evolution. Large-scale galaxy surveys are hindered by the presence of non-linearities which affect the luminous baryonic matter on small scales and have to rely on assumptions about (or deductions of) the relationship between luminous matter and mass distribution on large scales [11, 12, 13]. Supernova studies are afflicted by complicated systematics [14]. Hence, weak lensing can considerably help in the determination of poorly constrained parameters linked to the matter evolution and the moderate-redshift evolution of the redshift-distance relation. The most significant parameter is the equation of state of dark energy, $w \equiv p / \rho c^{2}$, and its time evolution. As is now generally accepted, there is strong evidence from a variety of cosmological probes like supernovae [15] and the CMB [10] that a dark energy component makes up about $70 \%$ of the total energy density budget of our Universe but its precise nature and state remain a mystery. Answers are unlikely to come from particle physics and our hopes lie in cosmology.

A variety of deep, large sky weak lensing surveys are either ongoing, scheduled or planned in the foreseeable future (Deep Lens Survey [16], NOAO Deep survey [17], The CFHT Legacy survey [18], darkCAM on VISTA, The Panoramic Survey Telescope and Rapid response System (Pan-STARRS) [20], the Large Synoptic Survey Telescope 21] and SNAP [19]). If one can rigorously account for the various systematics (such as the seeing, calibration, anisotropic point spread functions, redshift uncertainties etc.) likely to permeate the data, such surveys can offer if not definite answers at least fundamental clues on the nature of the dark energy, which lensing surveys are beginning to constrain $w$ 22]. In combination with other data sets, weak lensing surveys data can further overcome intrinsic degeneracies characteristic of each of the cosmological probes and strengthen the constraints on our cosmological model. In particular, predictive studies have been done which explore the possible improvements for parameter estimation of considering the three dimensionality of weak lensing, where photometric redshifts are used to provide estimates in three dimensions of the weak lensing shear field. As shown by Ishak [23], cosmic complementarity and 3D lensing tomography bring uncertainties on the equation of state to the level of a few percent. The use of full distance information on weak lensing surveys, enabling a full 3D analysis rather than tomography, can further reduce statistical errors on cosmological parameters [24].

Besides reducing statistical errors, there are other reasons for wishing to have photometric redshifts: weak lensing studies have to deal with systematic errors which could dominate the error budget as surveys improve (see [24] and

* pgc@roe.ac.uk; ${ }^{\dagger}$ afh@roe.ac.uk; ${ }^{\ddagger}$ tdk@roe.ac.uk 
references therein). The most important of these is the redshift distribution of the sources but correlated source galaxy intrinsic ellipticities (to date still not well-known) and the effect of source clustering are likely to become a considerable nuisance in the future. A reasonably accurate knowledge of the redshift of the sources can help in understanding, quantifying and ultimately removing such systematics. Furthermore, since acquisition of photometric redshifts requires several images of each galaxy in different bands, there is scope for better shape measurement, independent lensing studies, and so on.

In the past, most of weak lensing analysis were essentially limited to $2 \mathrm{D}$ approaches due to the lack of, or imprecise, photometric redshift information about individual galaxies. Moreover, such studies were usually applied to small patches of the sky necessarily involving a flat-sky approximation (see e.g. 25, 26 and 4, 7] for an application to data). A useful exception to this early trend is the theoretical work by Stebbins [27] who explored the 2D curved sky by means of the tensor spherical harmonics formalism. But over the last few years or so, with the arrival of surveys with more accurate photometric redshift information and the prospect of them reaching wider areas of the sky, interest has steadily grown in exploring the inherently full-sky 3D information contained in the lensing observables (see e.g. [28, 29, 30]). The way of dealing with any source redshift information has thus evolved over the years from basically a black and white picture to a 'fabulous Technicolour' approach. Originally it was used as a way of determining the redshift distribution of the sources only. It then evolved to a so-called tomographic analysis, where the sources were divided up in slices at different redshifts and a 2D analysis was then performed on each one of the slices. This can be useful when trying to detect clusters of galaxies [30, 31, 32, 33]. As shown by Hu [34] at a statistical level such method also presents certain advantages depending on the parameters one is interested in retrieving. For instance, for the amplitude of the matter power spectrum there can be significant gains when a source population is split into two, but little is gained by further finer subdivisions. For $w$ the gains are much larger as the $2 \mathrm{D}$ analysis constraints are weak. It is worth noting here that other 2D routes have been explored in pursuit of cosmologically sensitive weak-lensing estimators such as the study of higher-order statistics (for bispectrum detections see 35, 36 ) but if one wants to place strong constraints on $w$ a 3D study is compulsory. Several theoretical studies [37, 38, 39, 40] investigated how the lensing equations can be inverted to extract the 3D gravitational potential directly, thus offering the possibility of reconstruction of the 3D mass density field from weak-lensing data. Only recently in Heavens [24], was a truly 3D spectral statistical analysis on the full-sky developed, in which the individual source redshifts were taken into consideration per se. In there, the attention was mainly focused upon understanding the amount of extra information on the cosmological parameters that can be gleaned from a 3D shear map, rather than on establishing a framework for 3D weak lensing studies. Motivated by the previous theoretical considerations and the upcoming experimental prospects, it is timely to develop in detail a description of the weak lensing observables on the full three dimensional sky.

A full-sky 3D study of the weak lensing observables needs to combine at its root both the 3D weak lensing characteristics and the full-sky formalism. The weak lensing effect can be observed as a local modification of the surface number density of background galaxies (the magnification) and a distortion of their shape (the shear). As has been pointed out repeatedly before, the weak shear components present striking similarities with the Stokes parameters $Q$ and $U$ describing the linear polarisation of the CMB light, the major difference being its additional radial dependence. Like them, they are not invariant under a rotation of the reference frame used to describe them but rather transform as rank-2 tensors. One can then legitimately extend to the third radial dimension past work done for the full-sky polarisation of the CMB [41, 42, 43, 44, 45] and adapt it to the weak lensing analysis. In this work, we develop in more detail Heavens' original 3D full-sky analysis 24 weaving into our approach both the CMB polarisation formalism and past weak lensing theoretical 2D studies [25, 26, 27]. We use a spectral decomposition as it allows one to restrict the analysis to, for example, the linear or mildly non-linear regime and rely on the spin-weighted formalism, originally developed by Newman and Penrose in the 1960s [46].

This paper is organised as follows. In section [I] we review the main results of the theory of gravitational weak lensing which we generalise to 3 dimensions so that the relevant theoretical expressions depend explicitly on the radial distance from us. In subsection $\amalg B$ we start by presenting the weak lensing theoretical expressions in the familiar tensorial formalism, both in the full-sky and in the flat-sky approximation. In subsection ПI we introduce an alternative differential formalism we name the edth formalism, which encompasses the full and the flat-sky geometries. Such a mathematical apparatus reveals itself to be extremely convenient when working on the full-sky and has been extensively used in studies of the CMB polarisation but which we develop here for 3D weak-lensing fields. In section we spectrally decompose in the 3D full-sky the (spin-weight 2) weak lensing shear and (scalar) convergence fields in terms of spin-weighted spherical harmonics and Bessel functions. We derive their expansion coefficients in function of the gravitational potential coefficients by making full use of the edth formalism. In subsections IVA and IVB we calculate the weak lensing 3D power spectra in the full-sky and derive small-angle limits for completeness. Finally in section $\nabla$ we present results for the 2-point correlation function of the 3D shear field. In the Appendixes, we review in more detail the notation, the mathematics and the conventions chosen of the spin-weight $s$ functions and of the associated geometrical spin raising and lowering ( $\check{\partial}$ and $\bar{\partial})$ operators defined over any two-dimensional Riemannian 
manifold.

\section{WEAK LENSING THEORY IN 3D}

\section{A. The lensing potential}

In the linear regime, many scalar fields on the sky that are associated with large-scale structures can be interpreted as line-of-sight integrations of functions of the gravitational potential $\Phi$ with a given weight. A few examples of such scalar fields are the Integrated Sachs-Wolfe effect [4] or the Ostriker-Vishniac effect [8] imprinting themselves on the $\mathrm{CMB}$ and the gravitational weak lensing by cosmological structure (for a comprehensive review see [1]). For the gravitational weak lensing case, one can associate the so-called weak lensing potential $\phi$ for a given source at a 3D position in comoving space $\boldsymbol{r} \equiv(r, \theta, \varphi)$ to the peculiar gravitational potential $\Phi$ defined along the line-of-sight via (see [1])

$$
\phi(\boldsymbol{r})=\phi(r, \theta, \varphi)=\frac{2}{c^{2}} \int_{0}^{r} d r^{\prime} \frac{f_{K}\left(r-r^{\prime}\right)}{f_{K}(r) f_{K}\left(r^{\prime}\right)} \Phi\left(r^{\prime}, \theta, \varphi\right)
$$

where the Born approximation was assumed (i.e. the path of the integration, corresponding to the path of the photons emitted by the source, is assumed to be unperturbed by the lens). Although usually the lensing potential $\phi$ is regarded as a $2 \mathrm{D}$ radial projection of the $3 \mathrm{D}$ gravitational potential, it is in reality a $3 \mathrm{D}$ quantity. It is customary to average over the redshift distribution of the source galaxies, but this is not necessary if one has distance information about the individual sources. Here and in the remainder of the manuscript, bold letters denote 3-dimensional vectors, $c$ is the speed of light, $r \equiv r(t)$ is the comoving distance of the source at instant $t$ from the observer at the origin $(r=0)$ and $f_{K}(r) d \psi$ is the comoving transverse dimensionless separation for points separated by $d \psi$. We have $f_{K}(r)=\sin r, r$ and $\sinh r$ for a closed $(k=1)$, flat $(k=0)$ and open $(k=-1)$ universes respectively. The gravitational potential $\Phi$ is related to the underlying overdensity field $\delta(\boldsymbol{r}) \equiv \delta \rho(\boldsymbol{r}) / \bar{\rho}$ by the Poisson equation

$$
\nabla_{r}^{2} \Phi(\boldsymbol{r})=\frac{3 \Omega_{m} H_{0}^{2}}{2 a(t)} \delta(\boldsymbol{r})
$$

where the 3D gradient $\nabla_{r}$ is defined relative to comoving coordinates, $\Omega_{m}$ is the present-day total matter density, $H_{0}$ is the Hubble constant today in units of $\mathrm{km} / \mathrm{s} / \mathrm{Mpc}$ and $a(t)=1 /(1+z)$ is the scale factor. We use the comoving gauge or total-matter gauge (see e.g. [49, 50]).

If one wants to make a spectral expansion, such as a Fourier transform, then immediately one has a subtlety to consider. The lensing potential field, indirectly observed through its effect on the shapes of lensed galaxies, is not homogeneous, as it is viewed on the past light cone of the galaxies, and is a function of the gravitational potential which evolves with cosmic epoch. When referring to the transform of a field, such as the gravitational potential, at a lensed galaxy at distance $r$, we will mean the transform of the homogeneous field existing everywhere at the cosmic epoch corresponding to the time the observed light left the distant galaxy. Thus the coefficients of the expansion depend on the lookback time of the observation, and hence, rather paradoxically, on the distance $r$ itself.

There is a natural choice of basis functions to use for an expansion. Spherical coordinates are natural for various reasons, partly because the lensing potential is a radial integral, partly because partial sky coverage is more easily dealt with, as demonstrated in large-scale structure studies by [51]. In addition, errors in distances (from using photometric redshifts as distance indicators, for instance) are radial errors. The first of these means the coefficients of the expansion of the gravitational potential and of the lensing potential are straightforwardly related. The choice of basis functions is motivated by Poisson's equation (2). It makes sense to use the eigenfunctions of the Laplacian operator, since then the coefficients of the gravitational potential and of the density field are closely related (essentially by a factor $-k^{-2}$ ). In Cartesian coordinates, the eigenfunctions are the familiar exponential functions of Fourier analysis. If however the Laplacian is written in spherical coordinates $(r, \theta, \varphi)$, then in flat space the eigenfunctions become products of spherical harmonics and spherical Bessel functions: $Y_{\ell m}(\theta, \varphi) j_{\ell}(k r)$, with eigenvalue $-k^{2}$. So for scalar fields $f(\boldsymbol{r})$ in a flat background geometry, the natural 3D expansion is

$$
f_{\ell m}(k) \equiv \sqrt{\frac{2}{\pi}} \int d^{3} r f(\boldsymbol{r}) k j_{\ell}(k r) Y_{\ell m}^{*}(\theta, \varphi),
$$

where the numerical factor and the presence of $k$ are chosen for convenience. The inverse transform is

$$
f(\boldsymbol{r})=\sqrt{\frac{2}{\pi}} \int k d k \sum_{\ell=0}^{\infty} \sum_{m=-\ell}^{\ell} f_{\ell m}(k) j_{\ell}(k r) Y_{\ell m}(\theta, \varphi) .
$$


This is readily obtained from the orthonormality of the spherical harmonics and the orthogonality of the spherical Bessel functions (see Eqs. (B2) and (B5)).

Using this 3-dimensional expansion, one can relate the transform of the lensing potential to the gravitational potential field in a flat geometry [24] by

$$
\phi_{\ell m}(k)=\frac{4 k}{\pi c^{2}} \int_{0}^{\infty} d k^{\prime} k^{\prime} \int_{0}^{\infty} d r r j_{\ell}(k r) \int_{0}^{r} d r^{\prime}\left[\frac{r-r^{\prime}}{r^{\prime}}\right] j_{\ell}\left(k^{\prime} r^{\prime}\right) \Phi_{\ell m}\left(k^{\prime} ; r^{\prime}\right) .
$$

It is worth remarking that our expression differs from Eqs. (6) and (7) found in Heavens [24] due to a different choice of the 3D expansion conventions. One can further relate $\Phi_{\ell m}(k ; r)$ to the expansion of the matter overdensity $\delta_{\ell m}(k ; r)$ by using the Poisson equation:

$$
\Phi_{\ell m}(k ; r)=-\frac{3 \Omega_{m} H_{0}^{2}}{2 k^{2} a(r)} \delta_{\ell m}(k ; r)
$$

where here we put the time dependence (see discussion above) in the coefficients of the gravitational potential and matter overdensity fields by explicitly writing the coefficients as functions of the distance $r$ in addition to $\ell, m$ and $k$. We point out that the expansion coefficients of the lensing potential $\phi$ do not have the time dependence explicitly shown because $\phi$ is not by definition a homogeneous and isotropic field in $3 \mathrm{D}$ space.

These equations establish the relations between the scalar fields $\phi, \Phi$ and $\delta$. Since the statistical properties of $\delta$ are known for a given cosmological model, this opens up the possibility of using weak lensing in 3D (via $\phi$ ) for estimation of cosmological parameters. This would normally be done using the weak lensing shear field, $\gamma(\boldsymbol{r})$, which is not a scalar field, but rather a spin-weight 2 field. We will consider the statistics of this observable field in Section III.

\section{B. Weak lensing and the gravitational potential in the tensorial formalism}

In this subsection we review the main results of the theory of gravitational weak lensing in the tensorial formalism on the 3 dimensional spherical sky. In addition, we introduce the relevant formulae in the 3 dimensional flat-sky approximation for completeness.

\section{Weak lensing on the full-sky}

The 2 dimensional distortion of images of distant sources, located at a certain 3D comoving position in space $\boldsymbol{r}$, caused by the weak gravitational lensing by intervening structures is given by [1, 42, 52, 53.

$$
\left[\nabla_{i} \nabla_{j}-\frac{1}{2} g_{i j} \nabla^{2}\right] \phi(\boldsymbol{r})=\left[\gamma_{1}(\boldsymbol{r}) \sigma_{3}+\gamma_{2}(\boldsymbol{r}) \sigma_{1}\right]_{i j}
$$

where the indices $i, j \equiv(\theta, \varphi)$ stand for the polar $2 \mathrm{D}$ coordinate indices on the sphere, $\sigma_{1}$ and $\sigma_{3}$ are the Pauli matrices defined on the 2D spherical sky, $g_{i j}$ is the 2D metric on that surface given by $g=\operatorname{diag}\left(1, \sin ^{2} \theta\right)$ and $\phi$ is the lensing potential related to the gravitational potential $\Phi$ by Eq. (11). Here, and throughout, $\gamma_{1}$ and $\gamma_{2}$ are the components of the weak lensing shear produced by the gravitational tidal field, and which may be conveniently written as a complex shear $\gamma(\boldsymbol{r}) \equiv \gamma_{1}(\boldsymbol{r})+i \gamma_{2}(\boldsymbol{r})$. They correspond to the two orthogonal modes of the distortion which are, a priori, measurable on the sky with respect to a chosen fixed coordinate system. At fixed $r$, comparison of $\gamma$ at different locations $(\theta, \varphi)$ on the sphere is not possible. In curved space, comparisons are only possible locally, and, as $\gamma$ is not a scalar, parallel-transport of $\gamma$ from place to place is path-dependent. This fact lies at the source of the interest in first developing rotationally invariant $\mathrm{CMB}$ polarisation components in terms of electric and magnetic components [41] as we will see shortly in the context of weak lensing. The differential operator $\nabla$ corresponds to a $2 \mathrm{D}$ covariant derivative on the sphere of radius $r$, which for a scalar is given by the partial derivative $\nabla_{i}=\partial_{i}$, for a covariant vector $X_{i}$ (i.e. rank-1 covariant tensor) is given by $\nabla_{j} X_{i}=\partial_{j} X_{i}-\Gamma_{j i}^{k} X_{k}$ and for a contravariant vector $X^{i}$ is given by $\nabla_{j} X^{i}=\partial_{j} X^{i}+\Gamma_{k j}^{i} X^{k}$ with the Christoffel symbol $\Gamma_{j i}^{k}$ depending on the metric $g_{i j}$ (see for instance [54]). In our case, for which $g=\operatorname{diag}\left(1, \sin ^{2} \theta\right)$, we will have only three non-zero Christoffel symbols which are $\Gamma_{11}^{0}=-\sin \theta \cos \theta$ and $\Gamma_{01}^{1}=\Gamma_{10}^{1}=\cot \theta$. By construction, the Christoffel symbol is always symmetric in its lower indices $\Gamma_{j i}^{k}=\Gamma_{i j}^{k}$. Due to this symmetry property, there is an important result for scalar fields $X$ which is that $\nabla_{i} \nabla_{k} X=\nabla_{k} \nabla_{i} X$. In the remaining of this work we will apply this property to the lensing potential $\phi$. 
The lensing potential $\phi$ can alternatively be observed by means of the magnification via the isotropic convergence scalar field $\kappa$ defined as

$$
[\kappa(\boldsymbol{r})]_{i j}=\kappa(\boldsymbol{r}) I_{i j}=\frac{1}{2} g_{i j} \nabla^{2} \phi(\boldsymbol{r})
$$

where $\nabla^{2}=\nabla_{i} \nabla^{i}$ and $I_{i j}$ is the Identity matrix. We name $[\kappa]_{i j}$ the convergence field tensor. Note that the weak lensing regime corresponds to lensing configurations such that $|\kappa| \ll 1$ and $|\gamma| \ll 1$. In this specific regime, the magnification and the distortion of galaxy sources are so small that one cannot measure them individually, rather one needs to perform a statistical study of the lensed population.

It will be useful later to have the expressions (7) and (8) explicitly expanded in terms of covariant derivatives on the 2D spherical sky. Transforming from the Cartesian $\left\{\hat{\boldsymbol{e}}_{\boldsymbol{x}}(\hat{\boldsymbol{n}}), \hat{\boldsymbol{e}}_{\boldsymbol{y}}(\hat{\boldsymbol{n}})\right\}$ to a spherical polar 2-dimensional coordinate system $\left\{\hat{\boldsymbol{e}}_{\boldsymbol{\theta}}(\hat{\boldsymbol{n}}), \hat{\boldsymbol{e}}_{\boldsymbol{\varphi}}(\hat{\boldsymbol{n}})\right\}$, where we choose the basis of the two coordinate systems to be aligned such that $d x=d \theta$ and $d y=\sin \theta d \varphi$, we can explicitly express Eq. (7) for the weak lensing shear as

$$
[\gamma(\boldsymbol{r})]_{i j}=\left(\begin{array}{cc}
\gamma_{1}(\boldsymbol{r}) & \sin \theta \gamma_{2}(\boldsymbol{r}) \\
\sin \theta \gamma_{2}(\boldsymbol{r}) & -\sin ^{2} \theta \gamma_{1}(\boldsymbol{r})
\end{array}\right)=\left(\begin{array}{cc}
\frac{1}{2}\left[\nabla_{\theta} \nabla_{\theta}-\csc ^{2} \theta \nabla_{\varphi} \nabla_{\varphi}\right] & \nabla_{\varphi} \nabla_{\theta} \\
\nabla_{\varphi} \nabla_{\theta} & \frac{1}{2}\left[\nabla_{\varphi} \nabla_{\varphi}-\sin ^{2} \theta \nabla_{\theta} \nabla_{\theta}\right]
\end{array}\right) \phi(\boldsymbol{r}) .
$$

$[\gamma]_{i j}$ is the $2 \times 2$ symmetric and traceless cosmic shear tensor field associated to a source at a 3D position $\boldsymbol{r}$ defined on the sky and again $\nabla_{i}$ corresponds to a $2 \mathrm{D}$ covariant derivative on the sphere. We used the general result for scalar fields $\nabla_{\theta} \nabla_{\varphi} \phi=\nabla_{\varphi} \nabla_{\theta} \phi$. The $\sin \theta$ terms in the weak lensing shear tensor $[\gamma]_{i j}$ appear because the orthogonal $(\theta, \varphi)$ basis is not orthonormal. This result has been repeatedly used when describing the polarisation tensor $\mathcal{P}_{i j}$ of the CMB on the full-sky (see for example [55]). One can extract from Eq. (9) the individual components of the weak lensing shear $\gamma_{1}$ and $\gamma_{2}$

$$
\begin{aligned}
\gamma_{1}(\boldsymbol{r}) & =\frac{1}{2}\left[\nabla_{\theta} \nabla_{\theta}-\csc ^{2} \theta \nabla_{\varphi} \nabla_{\varphi}\right] \phi(\boldsymbol{r}), \\
\gamma_{2}(\boldsymbol{r}) & =\csc \theta \nabla_{\varphi} \nabla_{\theta} \phi(\boldsymbol{r}) .
\end{aligned}
$$

Similarly, one obtains for the convergence field tensor $[\kappa]_{i j}$ given by Eq. (8) the following expanded expression

$$
[\kappa(\boldsymbol{r})]_{i j}=\left(\begin{array}{cc}
1 & 0 \\
0 & \sin ^{2} \theta
\end{array}\right) \kappa(\boldsymbol{r})=\left(\begin{array}{cc}
\frac{1}{2}\left[\nabla_{\theta} \nabla_{\theta}+\csc ^{2} \theta \nabla_{\varphi} \nabla_{\varphi}\right] & 0 \\
0 & \frac{1}{2}\left[\nabla_{\varphi} \nabla_{\varphi}+\sin ^{2} \theta \nabla_{\theta} \nabla_{\theta}\right]
\end{array}\right) \phi(\boldsymbol{r})
$$

where $\kappa$, the scalar convergence field in the $3 \mathrm{D}$ full-sky, is given by

$$
\kappa(\boldsymbol{r})=\frac{1}{2}\left[\nabla_{\theta} \nabla_{\theta}+\csc ^{2} \theta \nabla_{\varphi} \nabla_{\varphi}\right] \phi(\boldsymbol{r}) .
$$

\section{Weak lensing on the flat-sky}

When analysing data on a small patch of the sky, one can use the flat-sky, or small-angle, approximation. There, one defines the standard Cartesian coordinate system $\left\{\hat{\boldsymbol{e}}_{\boldsymbol{x}}(\hat{\boldsymbol{n}}), \hat{\boldsymbol{e}}_{\boldsymbol{y}}(\hat{\boldsymbol{n}})\right\}$ with metric given by $g=\operatorname{diag}(1,1)$. The covariant derivatives simplify to the standard partial differentiation operators $\nabla_{i} \rightarrow \partial_{i}$. The above Eq. (9) then reduces to [26]

$$
[\gamma(\boldsymbol{r})]_{i j}=\left(\begin{array}{cc}
\gamma_{1}(\boldsymbol{r}) & \gamma_{2}(\boldsymbol{r}) \\
\gamma_{2}(\boldsymbol{r}) & -\gamma_{1}(\boldsymbol{r})
\end{array}\right)=\left(\partial_{i} \partial_{j}-\frac{1}{2} \delta_{i j} \nabla^{2}\right) \phi(\boldsymbol{r})
$$

in such a way that we recover the well-known expressions for the components $\gamma_{1}$ and $\gamma_{2}$ of the shear on the 3D flat-sky

$$
\begin{aligned}
\gamma_{1}(\boldsymbol{r}) & =\frac{1}{2}\left(\partial_{x}^{2}-\partial_{y}^{2}\right) \phi(\boldsymbol{r}) \\
\gamma_{2}(\boldsymbol{r}) & =\partial_{x} \partial_{y} \phi(\boldsymbol{r})
\end{aligned}
$$

The convergence field takes the form

$$
\kappa(\boldsymbol{r})=\frac{1}{2} \nabla^{2} \phi(\boldsymbol{r})=\frac{1}{2}\left(\partial_{x}^{2}+\partial_{y}^{2}\right) \phi(\boldsymbol{r})
$$

where $\nabla^{2}=\partial_{i} \partial^{i}=\partial_{i} \partial_{i}$ as the metric is given by $g=\operatorname{diag}(1,1)$. 


\section{Weak lensing shear as a spin weight 2 object}

Weak lensing induces a complex shear field $\gamma(\boldsymbol{r})$ which transforms under a rotation (by an angle $\psi$ in the anticlockwise direction in our conventions) of the fixed coordinate system according to $\gamma \rightarrow \gamma e^{-i s \psi}$, where $s=2$ is its spin weight. This phase dependence expresses the fact that the complex shear field $\gamma(\boldsymbol{r})$ is invariant under a rotation over $\pi$ radians. Also, the two components of the shear field, $\gamma_{1}$ and $\gamma_{2}$, are related by a $\pi / 4$ radians rotation which transforms one field into the other: $\gamma_{1}^{\prime}=-\gamma_{2}$ and $\gamma_{2}^{\prime}=\gamma_{1}$ where the prime denotes the transformed fields. In this subsection we use a description of the field based on a geometrical differential operator called edth and symbolised by $\precsim$. This operator was firstly introduced by Newman and Penrose in 1966 [46] (see also [56]) on the surface of the sphere in order to define the now widely used 'spin-weight $s$ spherical harmonics' which live on the 2D spherical space. A spin-weight $s$ spherical harmonic, symbolised as ${ }_{s} Y_{\ell m}$, can be seen as a generalisation of the scalar, vector and tensor spherical harmonics (see [57, 58]). The ${ }_{s} Y_{\ell m}$ (not defined for $|s|>l$ ) form a complete orthonormal basis for each $s$, a result that we will use later in order to describe the spin-weight $s$ functions on the 2D sphere. The spin $s$ of a function is related to its transformation properties under a rotation of the frame field where it is defined (for example a scalar is a spin-0 function). The $\partial$ (and its complex conjugate $\bar{\partial}$ ) act as raising (and lowering) operators on the 'quantum number' $s$ (integral number), such that ${ }_{s} Y_{\ell m}$ are eigenfunctions of $\delta \bar{\delta}$ and can be obtained by applying $\partial$ to the standard spin-weight 0 spherical harmonics $Y_{\ell m}$. The operator $\partial$ is effectively a covariant differentiation operator acting on the surface of the sphere which enables one to relate quantities of different spin. In particular, it allows one to conveniently relate spin-weight $s$ objects, which are not invariant under rotations of the coordinate frame, to scalar quantities, which are invariant quantities under rotations. All invariant differential operators on the sphere may be expressed in terms of it.

The operator $\widetilde{\partial}$ and the concept of spin-weight $\mathrm{s}$ functions have been generalised to any 2D Riemannian manifold (a 2D manifold with a metric) (e.g. [43, 59]). For details and further references see the Appendix, in particular Eq. (A5) for the expressions relating the $\delta$ operator to covariant derivatives in a 2D Riemannian manifold.

As pointed out originally by Newman \& Penrose [46], any spin-weight $s$ function defined on a 2D Riemannian manifold can be uniquely decomposed into a scalar gradient (or electric/even) 'E'-component and a scalar curl (or magnetic/odd) 'B'-component. We say that the spin-weight $s$ field $\eta$ is even if $\eta=\partial^{s} f$ and odd if $\eta=i \bar{\partial}^{s} f$ for some real-valued spin-weight 0 function $f$ (if $s<0$ then we interpret $\check{\partial}^{s}$ as $(-\bar{\partial})^{|s|}$ ). For $s=1$ this decomposition corresponds exactly to the classical Hodge-Helmholtz decomposition of a vector field into the sum of a gradient and a curl component.

As a spin-weight 2 object, the shear field can be written as the second edth-derivative of a complex potential 25 , 26, 27, 41, 46, 60, 63]:

$$
\begin{gathered}
\gamma(\boldsymbol{r}) \equiv \gamma_{1}(\boldsymbol{r})+i \gamma_{2}(\boldsymbol{r})=\frac{1}{2} \partial \partial\left[\phi_{E}(\boldsymbol{r})+i \phi_{B}(\boldsymbol{r})\right], \\
\gamma^{*}(\boldsymbol{r}) \equiv \gamma_{1}(\boldsymbol{r})-i \gamma_{2}(\boldsymbol{r})=\frac{1}{2} \bar{\partial} \bar{\partial}\left[\phi_{E}(\boldsymbol{r})-i \phi_{B}(\boldsymbol{r})\right]
\end{gathered}
$$

where we introduce two scalar real functions $\phi_{E}(\boldsymbol{r})$ and $\phi_{B}(\boldsymbol{r})$ for the even and odd parts. The normalisation factor of 2 was chosen so that one can later identify immediately the lensing potential to the even field $\phi_{E}(\boldsymbol{r})$. As we will see, weak shear is derivable from a real (lensing) potential $\phi_{E}(\boldsymbol{r})=\phi(\boldsymbol{r})$, requiring $\phi_{B}(\boldsymbol{r})=0$. This definition differs from the convention used in CMB polarisation studies (see for instance [43, 44]) and comes about due to the conventions adopted for the spin-weight formalism (see the Appendix A 1). Note that the scalar functions $\phi_{E}$ and $\phi_{B}$ introduced are invariant under rotations of the reference frame.

We emphasise that the spin-raising and lowering operators $ð$ and $\bar{\partial}$ act on the 2D manifold at a distance $r$. We are interested in their expressions both in the full-sky 2D sphere and in the flat-sky 2D Euclidean space. Their derivation in both geometries for any value of spin $s$ is detailed in the Appendices A2 and A3 and we give the final expressions for $\bar{\partial}$ and $\bar{\partial}$ here. In the 2D spherical full-sky we have

$$
\begin{aligned}
\partial_{s} f(\theta, \varphi) & =-\left(\partial_{\theta}+i \csc \theta \partial_{\varphi}-s \cot \theta\right)_{s} f(\theta, \varphi) \\
& =-\sin ^{s} \theta\left(\partial_{\theta}+i \csc \theta \partial_{\varphi}\right) \sin ^{-s} \theta_{s} f(\theta, \varphi),
\end{aligned}
$$

and

$$
\begin{aligned}
\bar{\partial}_{s} f(\theta, \varphi) & =-\left(\partial_{\theta}-i \csc \theta \partial_{\varphi}+s \cot \theta\right){ }_{s} f(\theta, \varphi) \\
& =-\sin ^{-s} \theta\left(\partial_{\theta}-i \csc \theta \partial_{\varphi}\right) \sin ^{s} \theta_{s} f(\theta, \varphi) .
\end{aligned}
$$

In the $2 \mathrm{D}$ flat-sky the latter reduce to

$$
\begin{aligned}
& \partial_{s} f(x, y)=-\left(\partial_{x}+i \partial_{y}\right)_{s} f(x, y), \\
& \bar{\partial}_{s} f(x, y)=-\left(\partial_{x}-i \partial_{y}\right)_{s} f(x, y) .
\end{aligned}
$$


If we use these results, or rather these results expressed in terms of covariant derivatives, Eq. (16) can be re-expressed explicitly in terms of covariant derivatives on the sphere and in the tensorial formalism used in section [1

$$
\begin{aligned}
{[\gamma(\boldsymbol{r})]_{i j}=\left(\begin{array}{cc}
\gamma_{1}(\boldsymbol{r}) & \sin \theta \gamma_{2}(\boldsymbol{r}) \\
\sin \theta \gamma_{2}(\boldsymbol{r}) & -\sin ^{2} \theta \gamma_{1}(\boldsymbol{r})
\end{array}\right) } & =\left(\begin{array}{cc}
\frac{1}{2}\left[\nabla_{\theta} \nabla_{\theta}-\csc ^{2} \theta \nabla_{\varphi} \nabla_{\varphi}\right] & \nabla_{\varphi} \nabla_{\theta} \\
\nabla_{\varphi} \nabla_{\theta} & \frac{1}{2}\left[\nabla_{\varphi} \nabla_{\varphi}-\sin ^{2} \theta \nabla_{\theta} \nabla_{\theta}\right]
\end{array}\right) \phi_{E}(\boldsymbol{r}) \\
& +\left(\begin{array}{cc}
-\csc \theta \nabla_{\varphi} \nabla_{\theta} & \frac{1}{2}\left[\begin{array}{c}
\left.\sin \theta \nabla_{\theta} \nabla_{\theta}-\csc \theta \nabla_{\varphi} \nabla_{\varphi}\right] \\
\frac{1}{2}\left[\sin \theta \nabla_{\theta} \nabla_{\theta}-\csc \theta \nabla_{\varphi} \nabla_{\varphi}\right]
\end{array}\right. \\
\sin \theta \nabla_{\varphi} \nabla_{\theta}
\end{array}\right) \phi_{B}(\boldsymbol{r})
\end{aligned}
$$

where we obeyed to the conventions defined in the Appendices A 1 and A2 In particular, we projected the complex cosmic shear $\gamma$ into a tensor $[\gamma]_{i j}$ by means of relation Eq. (A3) and again used the general result $\nabla_{\theta} \nabla_{\varphi} \phi=\nabla_{\varphi} \nabla_{\theta} \phi$. We can now compare directly the general E-B decomposition of the shear Eq. (21) with the theoretical predictions for gravitational weak lensing shear on the 3D full-sky Eq (9). We can do this because the choice of the 2D polar coordinate system in both cases is the same. It is then mathematically straightforward to identify $\phi_{E}$ with the lensing potential $\phi$ such that $\phi_{E}(\boldsymbol{r})=\phi(\boldsymbol{r})$ and $\phi_{B}(\boldsymbol{r})=0$. Similarly one can make such an identification in the flat-sky approximation and re-express explicitly Eq. (16) in terms of partial derivatives on a 2D Euclidean space in the same tensorial formalism as above

$$
[\gamma(\boldsymbol{r})]_{i j}=\left(\partial_{i} \partial_{j}-\frac{1}{2} \delta_{i j} \nabla^{2}\right) \phi_{E}(\boldsymbol{r})+\frac{1}{2}\left(\varepsilon_{k j} \partial_{i} \partial_{k}+\varepsilon_{k i} \partial_{k} \partial_{j}\right) \phi_{B}(\boldsymbol{r})
$$

where $\varepsilon_{i j}$ is the anti-symmetric 2 -dimensional Levi-Civita tensor for which $\varepsilon_{00}=\varepsilon_{11}=0$ and $\varepsilon_{01}=-\varepsilon_{10}=1$ [26]. If we compare this equation with the weak lensing theoretical expression in the flat-sky limit Eq. (13), the identification between $\phi$ and $\phi_{E}$ is, as expected, immediate. Therefore the shear field induced by gravitational tidal fields only produces an E-pattern in cosmic shear maps. Such a result has been shown before [25, 26, 27, 64, 65] and has been applied to weak lensing data in the flat-sky limit [4, 5, 6, 7, 8]. It was to be expected as density (scalar) perturbations only produce $E$-type effects. Hence the decomposition of the weak lensing data into E and B components presents a clear advantage over the $\gamma_{1}$ and $\gamma_{2}$ decomposition. On the full-sky it allows one to isolate the effect caused by weak lensing and disentangle it from any non-lensing contributions like noise, foregrounds or systematics which should contribute similarly to both the E and the B modes. It is worth noting that both gravitational waves [27], multiple light lensing scattering effects and source clustering [60] may also induce B modes, but their levels are expected to be small. As we will mention shortly, one can have additional spurious curl-type effects caused by the observational pixelisation or by finite fields, due to a leakage between the E and the B modes [43, 44]. In such a situation the B-mode acts as a vital test for any non-gravitational signal in the data. Aside from such complications arising by finite fields and pixelisation, the $\mathrm{E}$ and $\mathrm{B}$ potentials have also the advantage of being rotationally invariant and so there are no ambiguities in their definition related to the rotation of the coordinate system on the sphere. Their interpretation is thus clearer than the one of the $\gamma_{1}$ and $\gamma_{2}$ decomposition over the whole sky.

Having established this correspondence in 3D, one can recast both Eq. (7) and Eq. (13), as the following valuable relation between the weak lensing shear $\gamma$ and the lensing potential in terms of the $\widetilde{\partial}$ and $\overline{\widetilde{\partial}}$ operators

$$
\begin{aligned}
& \gamma(\boldsymbol{r})=\frac{1}{2} \partial \partial \phi(\boldsymbol{r}), \\
& \gamma^{*}(\boldsymbol{r})=\frac{1}{2} \bar{\precsim} \bar{\partial} \phi(\boldsymbol{r})
\end{aligned}
$$

or, alternatively, the equivalent relation for the two orthogonal components of $\gamma$

$$
\begin{aligned}
& \gamma_{1}(\boldsymbol{r})=\frac{1}{4}(ð ð+\bar{ð} \bar{\delta}) \phi(\boldsymbol{r}),
\end{aligned}
$$

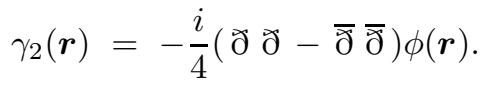

Similar expressions can be obtained for the scalar convergence field which is a spin-weight 0 object. As shown in the Eq. A8 of the Appendix, the Laplacian acting on a rank-0 tensor is equivalent to applying the combination $[\check{\partial}+\bar{\partial} \check{\partial}] / 2$ to the corresponding spin-weight 0 quantity. In particular, if one compares directly Eq. (A14) expressed in the full-sky to Eq. (12) one sees that Eq. (12) can equivalently be written as

$$
\kappa(\boldsymbol{r})=\frac{1}{4}[ð \bar{\partial}+\bar{ð} \precsim] \phi(\boldsymbol{r}) .
$$

Such an identification can also be performed in the flat-sky case. Note that there are natural extensions to higher

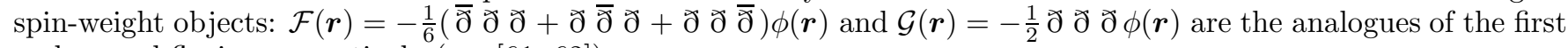
and second flexion respectively (see 61,62$]$ ). 
We now have both the weak lensing shear and convergence fields expressed in the edth formalism which encompasses both the full-sky and the flat-sky limits. Due to the role of the $\widetilde{\partial}(\bar{\partial})$ differential operators as spin-raising (lowering) the spin of spin-weight $s$ spherical harmonics, this alternative compact mathematical tool will be of particular beneficial use when studying weak lensing in the 3D full-sky.

\section{DECOMPOSITION OF 3D FULL-SKY WEAK LENSING INTO SPIN-WEIGHT SPHERICAL HARMONICS}

In the previous sections we have expressed the weak lensing shear and convergence fields as spin-weight functions. Aided by the edth formalism we have established their relation with the weak lensing potential by means of a simple and unique E-B decomposition scheme. In the present section, we concentrate on the 3D (geometrically flat) spherical sky where the previous fields can be decomposed into a combination of spin-weight spherical harmonics and Bessel functions. We thereby derive the relation between the expansion coefficients of the weak lensing quantities and the expansion coefficients of the weak lensing potential. The latter is readily related to the expansion coefficients of the gravitational potential and hence to those of the matter density field.

\section{A. Representation of a general distortion field in spin-weight spherical harmonics}

For spin-weight 2 fields such as the weak shear field, the natural 3D basis functions are products of radial functions and spin-weight 2 spherical harmonics. With this choice, the $3 \mathrm{D}$ expansion coefficients of $\gamma$ are related very simply to the expansion coefficients of the lensing potential, where the latter is expanded in products of ordinary (spin weight $0)$ spherical harmonics and the same radial functions.

Let us first assume a general distortion shear field $\eta(\boldsymbol{r})$. This field can be decomposed in two orthogonal components: $\eta(\boldsymbol{r}) \equiv \eta_{1}(\boldsymbol{r})+i \eta_{2}(\boldsymbol{r})$. Because of its transformation properties, such a field is a spin-weight 2 object. By consequent it can be decomposed into an even/'E' and an odd/'B' part by means of two scalar real functions defined on the 2D sphere of radius $r, \phi_{E}$ (for the even part) and $\phi_{B}$ (for the odd part) as in Eqs. (16) and (17)

$$
\eta(\boldsymbol{r})=\frac{1}{2} \partial \partial\left[\phi_{E}(\boldsymbol{r})+i \phi_{B}(\boldsymbol{r})\right], \quad \eta^{*}(\boldsymbol{r})=\frac{1}{2} \bar{\partial} \bar{\partial}\left[\phi_{E}(\boldsymbol{r})-i \phi_{B}(\boldsymbol{r})\right] .
$$

As discussed previously, the two real scalar functions $\phi_{E}$ and $\phi_{B}$ introduced completely characterise the distortion field. They have the advantage of being scalars which are invariant under any rotation of the coordinate system. We can then expand them in 3D on the full sky as we expanded the lensing potential field in Eq. (4) in terms of the standard (spin-0) spherical harmonics $Y_{\ell m}$ and of Bessel functions

$$
\begin{aligned}
& \phi_{E}(\boldsymbol{r})=-2 \int_{0}^{\infty} d k \sum_{\ell=0}^{\infty} \sum_{m=-\ell}^{\ell} \sqrt{\frac{(\ell-2) !}{(\ell+2) !}} a_{E, \ell m}(k) Z_{k \ell m}(r, \theta, \varphi), \\
& \phi_{B}(\boldsymbol{r})=-2 \int_{0}^{\infty} d k \sum_{\ell=0}^{\infty} \sum_{m=-\ell}^{\ell} \sqrt{\frac{(\ell-2) !}{(\ell+2) !}} a_{B, \ell m}(k) Z_{k \ell m}(r, \theta, \varphi)
\end{aligned}
$$

where we introduced the orthonormal basis functions $Z_{k \ell m}$ for simplicity and which, in a spatially flat background geometry, are given by

$$
Z_{k \ell m}(r, \theta, \varphi)=\sqrt{\frac{2}{\pi}} k j_{\ell}(k r) Y_{\ell m}(\theta, \varphi) .
$$

Apart from the normalisation factor of 2, the normalisation terms and the sign in Eqs. (28) were introduced to maintain consistency with previous works on CMB polarisation [4]]. The factor 2 is linked to the choice of the spin-weight conventions as established in the Appendix $\mathrm{A}$ and is necessary if one wants to recover relations between the expansion coefficients of $\gamma$ and of the fields $\phi_{E}$ and $\phi_{B}$ which are analogous to the relations between the CMB polarisation field $\mathcal{P}_{i j}$ and the electric and magnetic scalar fields [4]. We point out here that we do not assume 3D homogeneity and isotropy (at a fixed instant in time) for the scalar fields $\phi_{E}$ and $\phi_{B}$ and so do not introduce the time $r$ dependence in the coefficients of their expansion as discussed in section II A If we now substitute Eqs. (28) into Eqs. (27) and use the properties of the operators $\bar{\partial}$ and $\bar{\delta}$ acting on the standard (spin-weight 0) spherical harmonics (see Appendix 
Eqs. (B6)) one obtains naturally the following relations for $\eta$

$$
\begin{aligned}
\eta(\boldsymbol{r}) & =\int_{0}^{\infty} d k \sum_{\ell=0}^{\infty} \sum_{m=-\ell}^{\ell}{ }_{2} \eta_{\ell m}(k)_{2} Z_{k \ell m}(r, \theta, \varphi), \\
\eta^{*}(\boldsymbol{r}) & =\int_{0}^{\infty} d k \sum_{\ell=0}^{\infty} \sum_{m=-\ell}^{\ell}{ }_{-2} \eta_{\ell m}(k)_{-2} Z_{k \ell m}(r, \theta, \varphi)
\end{aligned}
$$

where the expansion coefficients are related to the $\phi_{E}$ and $\phi_{B}$ expansion coefficients by [41, 44]

$$
\begin{aligned}
{ }_{2} \eta_{\ell m}(k) & =-\left[a_{E, \ell m}+i a_{B, \ell m}\right](k), \quad{ }_{-2} \eta_{\ell m}(k)=-\left[a_{E, \ell m}-i a_{B, \ell m}\right](k) ; \\
a_{E, \ell m}(k) & =-\frac{1}{2}\left[{ }_{2} \eta_{\ell m}+{ }_{-2} \eta_{\ell m}\right](k), \quad a_{B, \ell m}(k)=\frac{i}{2}\left[{ }_{2} \eta_{\ell m}-{ }_{-2} \eta_{\ell m}\right](k) .
\end{aligned}
$$

and where now the orthonormal basis functions ${ }_{ \pm 2} Z_{k \ell m}$ are expressed in terms of a set of functions, the spin-weight \pm 2 spherical harmonics ${ }_{ \pm 2} Y_{\ell m}$, the ideal basis to express any spin-weight \pm 2 function

$$
{ }_{s} Z_{k \ell m}(r, \theta, \varphi)=\sqrt{\frac{2}{\pi}} k j_{\ell}(k r){ }_{s} Y_{\ell m}(\theta, \varphi)
$$

where the spin is given by $s= \pm 2$. As we can see, any distortion field $\eta$ defined on the 3D full-sky is most naturally expressed in terms of spin-weight 2 spherical harmonics, which define a set of orthonormal basis on the surface of the sky. The edth formalism introduced in the previous section has just shown its mathematical advantages when working in spherical space with spherical harmonics.

Inserting Eqs. (31) into Eqs. (30) we can also relate the $\eta_{1}$ and $\eta_{2}$ orthogonal components to the E-B decomposition expansion coefficients of the distortion field as

$$
\begin{aligned}
& \eta_{1}(\boldsymbol{r})=-\int_{0}^{\infty} d k \sum_{\ell=0}^{\infty} \sum_{m=-\ell}^{\ell}\left[a_{E, \ell m}(k) X_{1, k \ell m}(r, \theta, \varphi)+i a_{B, \ell m}(k) X_{2, k \ell m}(r, \theta, \varphi)\right], \\
& \eta_{2}(\boldsymbol{r})=-\int_{0}^{\infty} d k \sum_{\ell=0}^{\infty} \sum_{m=-\ell}^{\ell}\left[a_{B, \ell m}(k) X_{1, k \ell m}(r, \theta, \varphi)-i a_{E, \ell m}(k) X_{2, k \ell m}(r, \theta, \varphi)\right]
\end{aligned}
$$

where we defined two new basis sets: $X_{1, k \ell m}=\left({ }_{2} Z_{k \ell m}+{ }_{-2} Z_{k \ell m}\right) / 2$ and $X_{2, k \ell m}=\left({ }_{2} Z_{k \ell m}-{ }_{-2} Z_{k \ell m}\right) / 2$. As we have the relations $X_{1, k \ell m}^{*}=-X_{1, k-\ell m}, X_{2, k \ell m}^{*}=-X_{2, k-\ell m}, a_{E, \ell m}^{*}=a_{E,-\ell m}$ and $a_{B, \ell m}^{*}=a_{B,-\ell m}$, the coefficients $\gamma_{1}$ and $\gamma_{2}$ are real quantities.

Both representations can be chosen to study a general observed distortion field $\eta$, i.e. either use the $\eta_{1}$ and $\eta_{2}$ components or transform them into $E$ and $B$ type quantities. They contain the same information but, as explained before, the $E$ and $B$ are rotationally invariant on the full-sky and behave differently under parity transformation [42, 46]. In terms of the expansion coefficients, $a_{E, \ell m}(k)$ has parity $(-1)^{\ell}$ while $a_{B, \ell m}(k)$ has parity $(-1)^{\ell+1}$, a property which may certainly be useful when trying to characterise the origin of the distortion field observed on the sky. In particular, the E-B decomposition components have a special meaning in the context of weak lensing studies as weak lensing is in nature an E-type field and does not produce any B-type component, as we saw before in section!C

In practice, if one wants to determine the real scalar functions $\phi_{E}$ and $\phi_{B}$ describing a given distortion field on the sky one should use Eqs. (27). This is a trivial task if our observations are performed over the full-sky because one can use the orthogonality of the spin-weight spherical harmonics to obtain the $\mathrm{E}$ and $\mathrm{B}$ expansion coefficients from the measured field $\gamma$. But full-sky observations are unrealistic. Even if an experiment does cover the whole sky, certain regions always need to be removed in order to minimise the foreground contribution (such as bright stars). The perverse side effect of partial sky-coverage is to induce an E-B mode mixing. In this case the non-local decomposition (27) is not unique. If one tries to solve for $\phi_{E}$ or $\phi_{B}$ by taking linear combinations of second derivatives of the distortion field one gets

$$
\begin{aligned}
\nabla^{2}\left(\nabla^{2}+2\right) \phi_{E} & =\frac{1}{2}\left[\bar{\varnothing} \bar{\partial} \eta+ð \partial \eta^{*}\right], \\
\nabla^{2}\left(\nabla^{2}+2\right) \phi_{B} & =\frac{i}{2}\left[\bar{\varnothing} \bar{\partial} \eta-\partial \partial \eta^{*}\right] .
\end{aligned}
$$

This result enables one to use distortion observations to construct the $\mathrm{E}$ and $\mathrm{B}$ distortion shear modes on the full-sky. But over a cut-sky, solving this system implies the specification of unknown boundary conditions (i.e. value of the 
potentials and their derivatives at the boundaries). This problem has been extensively studied by many in the context of CMB polarisation measurements [43, 44] but to date separation of E-B modes has been performed statistically during the estimation of the power spectra using the so-called pseudo- $C_{\ell}$ method (see $[66]$ ). On the flat-sky and in the context of weak lensing, the locally defined aperture mass $M_{a p}$ may similarly provide unambiguous mode separation [67, 68]. A similar statistic can be built for the weak lensing B mode. It is beyond the scope of this work to enter into technical details related to mode separation, so we refer the reader to Challinor [58] for a useful recent CMB polarisation review and van Waerbeke and Mellier 2] for an updated weak lensing review.

\section{B. Representation of the weak lensing shear and convergence fields in spin-weight spherical harmonics}

The previous results can easily be applied to the case of the weak lensing shear field $\gamma$ for which (see Eqs. (23) and (24)

$$
\gamma(\boldsymbol{r})=\frac{1}{2} \partial ð \phi(\boldsymbol{r}), \gamma^{*}(\boldsymbol{r})=\frac{1}{2} \bar{\partial} \bar{\partial} \phi(\boldsymbol{r})
$$

where $\phi$ is the lensing potential defined in Eq. (11) and which we can expand in 3D as in Eq. (4) where the expansion coefficients are $\phi_{\ell m}(k)$. Comparison of the 3D expansion of the lensing potential $\phi$ with Eq. (28) shows that the $a_{E, \ell m}$ and the $\phi_{\ell m}$ are related by

$$
a_{E, \ell m}(k)=-\frac{1}{2} \sqrt{\frac{(\ell+2) !}{(\ell-2) !}} \phi_{\ell m}(k) .
$$

As demonstrated before, for gravitational weak lensing, $a_{B, \ell m}(k)=0$. The $3 \mathrm{D}$ full-sky coefficients of the spin-weight 2 shear field $\gamma,{ }_{2} \gamma_{\ell m}(k)$ and ${ }_{-2} \gamma_{\ell m}(k)$, defined similarly to Eq. (30) are then related to $\phi_{\ell m}$ by (see [24, 38, 42])

$$
{ }_{2} \gamma_{\ell m}(k)={ }_{-2} \gamma_{\ell m}(k)=\frac{1}{2} \sqrt{\frac{(\ell+2) !}{(\ell-2) !}} \phi_{\ell m}(k) .
$$

The standard components of the weak lensing shear $\gamma_{1}$ and $\gamma_{2}$ can be directly obtained from this last relation. If one uses the Eq. (34) with $a_{B, \ell m}(k)=0$ and the following expansion in 3D for the shear components $\gamma_{1}$ and $\gamma_{2}$

$$
\begin{aligned}
& \gamma_{1}(\boldsymbol{r})=\int_{0}^{\infty} d k \sum_{\ell=0}^{\infty} \sum_{m=-\ell}^{\ell} \gamma_{1, \ell m}(k) X_{1, k \ell m}(r, \theta, \varphi), \\
& \gamma_{2}(\boldsymbol{r})=\int_{0}^{\infty} d k \sum_{\ell=0}^{\infty} \sum_{m=-\ell}^{\ell} \gamma_{2, \ell m}(k) X_{2, k \ell m}(r, \theta, \varphi)
\end{aligned}
$$

where $X_{1, k \ell m}$ and $X_{2, k \ell m}$ were defined as in Eq. (34), one immediately has

$$
\gamma_{1, \ell m}(k)=i \gamma_{2, \ell m}(k)=-a_{E, \ell m}(k),
$$

where the expression for $a_{E, \ell m}$ in terms of the lensing potential was defined in Eq. (36). As we see, aside from the physical and experimental advantages of the $E-B$ decomposition scheme, using either of the expansion coefficients $\gamma_{1 / 2, \ell m}$ or $a_{E, \ell m}$ is equivalent as they are very simply related.

Likewise, we can do the same exercise for the convergence field $\kappa$. From Eq. (26) and using Eqs. (B6), the (scalar) expansion coefficients of $\kappa$ are given by [42]

$$
\kappa_{\ell m}(k)=-\frac{\ell(\ell+1)}{2} \phi_{\ell m}(k)
$$

To summarise, the coefficients of the expansion of the shear field in spin-weight 2 spherical harmonics and spherical Bessel functions, ${ }_{ \pm 2} \gamma_{\ell m}(k)$ are related to those of the lensing potential $\phi_{\ell m}(k)$ by Eq. (37). The $\phi_{\ell m}(k)$ are related to the gravitational potential $\Phi_{\ell m}(k)$ by Eq. (5), and these are in turn related to the overdensity field by Poisson's equation (6). In this way we establish a connection between the observable shear quantities ${ }_{{ }_{2}} \gamma_{\ell m}(k)$ (and associated quantities) and the overdensity field, whose statistical quantities have known dependence on cosmological parameters. In the knowledge of such results one may proceed to the derivation the 3D full-sky power spectra (or any any relevant statistics) of the weak lensing shear and convergence fields. 


\section{WEAK LENSING 3D POWER SPECTRA}

We shall now be interested in calculating the weak lensing shear $\gamma$ and convergence $\kappa$ 3D power spectra and in relating them to the $3 \mathrm{D}$ gravitational potential power spectra $C^{\Phi \Phi}$ both in the full-sky and flat-sky approximation.

\section{A. Weak lensing 3D power spectra on the full-sky}

If one performs a 3D spectral decomposition in the full-sky of a statistically homogeneous and isotropic field $f(\boldsymbol{r} ; r)$ at time instant defined by $r$ (one can use the comoving distance $r$ as a measure of real time $t$ as $r$ and $t$ are physically equivalent being related via the scale factor $a$ ) with coefficients $f_{\ell m}(k ; r)$ as in Eq. (3), then the 3D power spectrum of the field at time $r, C_{\ell}(k ; r)$, is defined by

$$
\left\langle f_{\ell m}(k ; r) f_{\ell^{\prime} m^{\prime}}^{*}\left(k^{\prime} ; r\right)\right\rangle=C_{\ell}(k ; r) \delta_{D}\left(k-k^{\prime}\right) \delta_{\ell \ell^{\prime}} \delta_{m m^{\prime}}
$$

where $\delta_{i j}$ is a Kronecker delta function and $\delta_{D}(x)$ a 1D Dirac delta function. The time dependence (introduced through $r$ ) of the coefficients, at which the spectral expansion is performed, was explained in section IA and is necessary to ensure the 3D spatial homogeneity (and isotropy) of the field we are expanding at a certain time instant. Most importantly, $C_{\ell}(k ; r)$ is independent of $\ell$, and is simply the 3D power spectrum $P(k ; r)$ :

$$
C_{\ell}(k ; r)=P(k ; r)
$$

where $P(k ; r)$ is obtained from performing a 3D Fourier expansion of the field $f(\boldsymbol{r} ; r)$ so that $\left\langle f(\boldsymbol{k} ; r) f^{*}\left(\boldsymbol{k}^{\prime} ; r\right)\right\rangle=$ $(2 \pi)^{3} P(k ; r) \delta_{D}^{3}\left(\boldsymbol{k}-\boldsymbol{k}^{\prime}\right)$. Indeed the spherical expansion in 3D of Eq. (4) for $f(\boldsymbol{r} ; r)$ is equivalent to the $3 \mathrm{D}$ Fourier expansion

$$
f(\boldsymbol{r} ; r)=\frac{1}{(2 \pi)^{3}} \int d^{3} \boldsymbol{k} f(\boldsymbol{k} ; r) e^{i \boldsymbol{k} \cdot \boldsymbol{r}}
$$

because of the well-known identity [49]

$$
e^{i \boldsymbol{k} \cdot \boldsymbol{r}}=4 \pi \sum_{\ell} \sum_{m=-\ell}^{m=\ell} i^{\ell} j_{\ell}(k r) Y_{\ell m}\left(\theta_{k}, \varphi_{k}\right) Y_{\ell m}\left(\theta_{r}, \varphi_{r}\right)
$$

One can see this by substituting Eq. (44) into Eq. (43) and using the identity $d^{3} k=k^{2} d \Omega_{k} d k$ where $\Omega_{k} \equiv\left(\theta_{k}, \varphi_{k}\right)$ so that we obtain the following relation between the expansion coefficients $f_{\ell m}(k ; r)$ (see Eq. (3) $)$ and $f(\boldsymbol{k} ; r)$ [49]

$$
f_{\ell m}(k ; r)=\frac{1}{\sqrt{8 \pi^{3}}} k i^{\ell} \int d \Omega_{k} f(\boldsymbol{k} ; r) Y_{\ell m}\left(\theta_{k}, \varphi_{k}\right) .
$$

One can then substitute this last expression in Eq. (41) and use $\left\langle f(\boldsymbol{k} ; r) f^{*}\left(\boldsymbol{k}^{\prime} ; r\right)\right\rangle=(2 \pi)^{3} P(k ; r) \delta_{D}^{3}\left(\boldsymbol{k}-\boldsymbol{k}^{\prime}\right)$. The trick then relies in replacing $\delta_{D}^{3}\left(\boldsymbol{k}-\boldsymbol{k}^{\prime}\right)=\int d^{3} x e^{i\left(\boldsymbol{k}-\boldsymbol{k}^{\prime}\right) \cdot \boldsymbol{x}} /(2 \pi)^{3}$ and using the identity (44). The orthogonality relations of the spherical harmonics (B2) and of the Bessel functions (B5) as well as $Y_{\ell m}^{*}=(-1)^{m} Y_{\ell-m}$ will then be useful to reach the final result, i.e. $C_{\ell}(k ; r)=P(k ; r)$.

Now if we use the expansion coefficients of the field located at different distances (or equivalently at different times from us) say at $r$ and $r^{\prime}$, to effectively calculate the cross-power spectrum of two different homogeneous and isotropic fields, $f(\boldsymbol{r} ; r)$ and $f\left(\boldsymbol{r}^{\prime} ; r^{\prime}\right)$, then one can still argue that the 3D homogeneity and isotropy argument holds:

$$
\left\langle a_{\ell m}(k ; r) a_{\ell^{\prime} m^{\prime}}^{*}\left(k^{\prime} ; r^{\prime}\right)\right\rangle=C_{\ell}\left(k ; r, r^{\prime}\right) \delta_{D}\left(k-k^{\prime}\right) \delta_{\ell \ell^{\prime}} \delta_{m m^{\prime}}
$$

where now we have the identity

$$
C_{\ell}\left(k ; r, r^{\prime}\right)=P\left(k ; r, r^{\prime}\right)
$$

where again $P\left(k ; r, r^{\prime}\right)$ is defined by $\left\langle f(\boldsymbol{k} ; r) f^{*}\left(\boldsymbol{k}^{\prime} ; r^{\prime}\right)\right\rangle=(2 \pi)^{3} P\left(k ; r, r^{\prime}\right) \delta_{D}^{3}\left(\boldsymbol{k}-\boldsymbol{k}^{\prime}\right)$. A similar result is frequently utilised to determine the cross-power spectra of different homogeneous and isotropic fields, like for instance the weak gravitational lensing of the CMB and the cosmic shear fields 53. It will be necessary to us when calculating the power spectra of the gravitational potential field $\Phi$ which comes about when calculating the spectra of the lensing potential. Again we stress that, contrary to the gravitational potential, the 3D lensing potential $\phi$ is not homogeneous and 
isotropic in $3 \mathrm{D}$ space. It is given by a $2 \mathrm{D}$ projection at each source distance $r$ of the gravitational potential existing between us and the source and so it maintains the homogeneity and isotropy characteristics of the gravitational potential field on the $2 \mathrm{D}$ sky, but not in the radial direction. We will then use for the field $\phi$ the relation

$$
\left\langle\phi_{\ell m}(k) \phi_{\ell^{\prime} m^{\prime}}^{*}\left(k^{\prime}\right)\right\rangle=C_{\ell}^{\phi \phi}\left(k, k^{\prime}\right) \delta_{\ell \ell^{\prime}} \delta_{m m^{\prime}},
$$

where $C_{\ell}^{\phi \phi}$ is the $3 \mathrm{D}$ lensing potential power spectrum. Contrary to the case of a 3D homogeneous and isotropic field, an equivalent relation to Eq. (42) does not hold any longer. Naturally, the lensing potential, the shear and the convergence all share the same statistical properties.

For completeness, although redundant, we present the various possible shear power spectra depending on the type of shear decomposition chosen. If one uses the expansion coefficients of the shear $\gamma(\boldsymbol{r})$ itself, i.e. ${ }_{ \pm 2} \gamma_{\ell m}(k ; r)$ of Eq. (37), then one gets

$$
C_{\ell}^{\gamma \gamma}\left(k_{1}, k_{2}\right)=\frac{1}{4} \frac{(\ell+2) !}{(\ell-2) !} C_{\ell}^{\phi \phi}\left(k_{1}, k_{2}\right)
$$

where $C_{\ell}^{\phi \phi}$ is the lensing potential power spectra. All the remaining power spectra can then be conveniently expressed in terms of $C_{\ell}^{\gamma \gamma}\left(k_{1}, k_{2}\right)$. For the $E$ - $B$ decomposition components, i.e. $a_{E, \ell m}(k)$ of Eq. (36) and $a_{B, \ell m}(k)$, one has [42]

$$
\begin{aligned}
& C_{\ell}^{E E}\left(k_{1}, k_{2}\right)=C_{\ell}^{\gamma \gamma}\left(k_{1}, k_{2}\right), \\
& C_{\ell}^{B B}\left(k_{1}, k_{2}\right)=0 .
\end{aligned}
$$

And finally for the $\gamma_{1}$ and $\gamma_{2}$ shear decomposition Eqs. (38) and (39) one obtains

$$
C_{\ell}^{\gamma_{1} \gamma_{1}}\left(k_{1}, k_{2}\right)=C_{\ell}^{\gamma_{2} \gamma_{2}}\left(k_{1}, k_{2}\right)=C_{\ell}^{\gamma \gamma}\left(k_{1}, k_{2}\right)
$$

where for symmetry reasons $C^{\gamma_{1} \gamma_{2}}=0$. Note that the expansions of $\gamma_{1}$ and $\gamma_{2}$ are in terms of the orthogonal but not orthonormal functions $X_{1, k \ell m}$ and $X_{2, k \ell m}$, so $\gamma_{1}$ and $\gamma_{2}$ each contribute half to the power of $\gamma$, as expected. Concerning the convergence field we have [42]

$$
C_{\ell}^{\kappa \kappa}\left(k_{1}, k_{2}\right)=\frac{\ell^{2}(\ell+1)^{2}}{4} C_{\ell}^{\phi \phi}\left(k_{1}, k_{2}\right)
$$

The next and final step is to relate the lensing and the gravitational potential power spectra, $C_{\ell}^{\phi \phi}$ and $C_{\ell}^{\Phi \Phi}$ respectively, by means of Eq. (5). After some straightforward algebra we obtain

$$
C_{\ell}^{\phi \phi}\left(k_{1}, k_{2}\right)=\frac{16}{\pi^{2} c^{4}} \int_{0}^{\infty} k^{2} d k I_{\ell}\left(k_{1}, k\right) I_{\ell}\left(k_{2}, k\right)
$$

with

$$
I_{\ell}\left(k_{i}, k\right) \equiv k_{i} \int_{0}^{\infty} d r r j_{\ell}\left(k_{i} r\right) \int_{0}^{r} d r^{\prime}\left[\frac{r-r^{\prime}}{r^{\prime}}\right] j_{\ell}\left(k r^{\prime}\right) \sqrt{P^{\Phi \Phi}\left(k ; r^{\prime}\right)}
$$

where we have used Eq. (45) applied to the gravitational potential field and introduced the familiar 3D power spectrum of the gravitational potential $P^{\Phi \Phi}$ via Eq. (46). For a non-uniform clustering of sources, the number density needs to be introduced into the outer integral 69]. The correlations in the potential field are significantly non-zero for small separations (much smaller than the speed of light times the timescale over which it evolves), so we have assumed for convenience that the gravitational potential power spectrum can be accurately approximated by

$$
P^{\Phi \Phi}\left(k ; r, r^{\prime}\right) \simeq \sqrt{P^{\Phi \Phi}(k ; r) P^{\Phi \Phi}\left(k ; r^{\prime}\right)} .
$$

This can be seen by noting that Poisson's equation implies that

$$
\Phi(\boldsymbol{k} ; r)=-\frac{3 \Omega_{m} H_{0}^{2}}{2 a(t) k^{2}} \delta(\boldsymbol{k} ; r)
$$

and so

$$
\left\langle\Phi(\boldsymbol{k} ; r) \Phi^{*}\left(\boldsymbol{k}^{\prime} ; r^{\prime}\right)\right\rangle=\left(\frac{3 \Omega_{m} H_{0}^{2}}{2}\right)^{2} \int \frac{d^{3} \boldsymbol{r} d^{3} \boldsymbol{r}^{\prime}}{a(t) a\left(t^{\prime}\right)} \frac{\left\langle\delta(\boldsymbol{k} ; r) \delta^{*}\left(\boldsymbol{k}^{\prime} ; r^{\prime}\right)\right\rangle}{k^{2} k^{\prime 2}} e^{-i \boldsymbol{k} \cdot \boldsymbol{r}+i \boldsymbol{k}^{\prime} \cdot \boldsymbol{r}^{\prime}}
$$


and the correlation of $\delta$ is restricted to small scales $\left|\boldsymbol{r}-\boldsymbol{r}^{\prime}\right| \leq 100 \mathrm{Mpc}$. The lookback time over such a distance is small, so we can approximate $\boldsymbol{r} \simeq \boldsymbol{r}^{\prime}$ (or $\left.t \simeq t^{\prime}\right)$. Therefore we can replace the power spectra $P^{\Phi \Phi}\left(k ; r, r^{\prime}\right)$ by either $P^{\Phi \Phi}(k ; r)$ or $P^{\Phi \Phi}\left(k ; r^{\prime}\right)$. For algebraic convenience, we choose the geometric mean of the power spectra, which allows us to separate two internal integrals and reduce computation time significantly. A further justification is apparent in Fig. (2): the Bessel functions cut off long-wavelength contributions with $k \leq \ell / r_{\max }$ where $r_{\max }$ is the extent of the survey. Practical issues of implementation are briefly discussed at the end of the next section. Note that the two-point statistics of the shear field are dependent on the nonlinear potential power spectrum, which is related to the nonlinear matter power spectrum via Poisson's equation. Accurate fits for the nonlinear matter power spectrum are available to enough wavenumbers [70], but the accuracy for high-precision determination of dark energy properties needs to be tested with simulations.

\section{B. Weak lensing 3D power spectra in the flat-sky limit}

In the flat-sky, we can expand a 3D field $f$ at 3D position $\boldsymbol{r} \equiv(r, \vec{\theta})$ on the sky into a combination of 2D Fourier modes and Bessel functions in the radial direction $r$

$$
\begin{aligned}
& f(r, \vec{\theta})=\sqrt{\frac{2}{\pi}} \int_{0}^{\infty} k d k \int_{0}^{\infty} \frac{d^{2} \vec{\ell}}{(2 \pi)^{2}} f(k, \vec{\ell}) j_{\ell}(k r) e^{i \vec{\ell} \cdot \vec{\theta}}, \\
& f(k, \vec{\ell})=\sqrt{\frac{2}{\pi}} \int_{0}^{\infty} r^{2} d r \int_{0}^{\infty} d^{2} \theta f(r, \vec{\theta}) k j_{\ell}(k r) e^{-i \vec{\ell} \cdot \vec{\theta}}
\end{aligned}
$$

where the notation $\vec{x}$ describes $2 \mathrm{D}$ vectors. Such an expansion was chosen to maintain a direct relation with the 3D full-sky expansion introduced previously. It thus presents the same attractive advantages as its full-sky counterpart (see discussion in section $\amalg A$ ). One can now easily obtain the full-sky to flat-sky correspondence, i.e. establish a relation between the expansion coefficients $f(k, \vec{\ell})$ in the flat-sky as defined above and $f_{\ell m}(k)$ in the full-sky defined in Eq. (4).

For small angles around the pole of spherical coordinates, defined by the angles $(\theta, \varphi)$ ignoring the radial dependence, for which $\theta \rightarrow 0$, one can use the following expansion of the plane 2D wave [42, 71]

$$
e^{i \vec{\ell} \cdot \vec{\theta}} \simeq \sqrt{\frac{2 \pi}{\ell}} \sum_{m} i^{m} Y_{\ell m}(\theta, \varphi) e^{-i m \varphi_{\ell}}
$$

where $\vec{\ell}=\left(\ell \cos \varphi_{\ell}, \ell \sin \varphi_{\ell}\right)$ and $\vec{\theta}=(\theta \cos \varphi, \theta \sin \varphi)$. The vector $\vec{\ell}$ can be interpreted as the continuous limit of the integer that labels the spherical harmonics $Y_{\ell m}$. Replacing Eq. (59) into Eq. (57) and making use of $\int_{0}^{\infty} d^{2} \vec{\ell}=$ $\int_{0}^{\infty} \ell d \ell \int_{0}^{2 \pi} d \varphi_{\ell} \rightarrow \sum_{l} \ell \int_{0}^{2 \pi} d \varphi_{\ell}$ for high $\ell$, one obtains the following relation between the 3D flat-sky and the 3D full-sky coefficients

$$
f_{\ell m}(k)=\sqrt{\frac{\ell}{2 \pi}} i^{m} \int_{0}^{2 \pi} \frac{d \varphi_{\ell}}{2 \pi} e^{-i m \varphi_{l}} f(k, \vec{\ell})
$$

The inverse relation is

$$
f(k, \vec{\ell})=\sqrt{\frac{2 \pi}{\ell}} \sum_{m} i^{-m} f_{\ell m}(k) e^{i m \varphi_{l}}
$$

We point out that, as expected, we recover the same relations that were obtained by others for the 2D case [42, 71], as the small-angle limit only affects the 2D angular expansion, not the radial direction. As the reader may have guessed, we also recover that the flat-sky $\mathcal{C}^{\Phi \Phi}$ and the full-sky $C^{\Phi \Phi}$ power spectra are equivalent in the high- $\ell$ limit. For a fully $3 \mathrm{D}$ homogeneous and isotropic field, like the gravitational potential we have

$$
\mathcal{C}^{\Phi \Phi}(k, l ; r) \stackrel{\ell \rightarrow \infty}{=} C_{\ell}^{\Phi \Phi}(k ; r)=P^{\Phi \Phi}(k ; r)
$$

where we have used Eq. (41), the definition $\left\langle\Phi(k, \vec{\ell}) \Phi^{*}\left(k^{\prime}, \overrightarrow{\ell^{\prime}}\right)\right\rangle=(2 \pi)^{2} \mathcal{C}^{\Phi \Phi}(k, \ell) \delta_{D}\left(k-k^{\prime}\right) \delta_{D}^{2}\left(\vec{\ell}-\overrightarrow{\ell^{\prime}}\right)$ and Eq. (42). Again $P^{\Phi \Phi}$ is the $3 \mathrm{D}$ gravitational potential power spectrum. For a $3 \mathrm{D}$ field which is homogeneous and isotropic only on the $2 \mathrm{D}$ sky (e.g. the lensing potential)

$$
\mathcal{C}^{\phi \phi}\left(k_{1}, k_{2}, l\right) \stackrel{\ell \rightarrow \infty}{=} C_{\ell}^{\phi \phi}\left(k_{1}, k_{2}\right)
$$


where in this case we have defined the power spectra via $\left\langle\phi(k, \vec{\ell}) \phi^{*}\left(k^{\prime}, \overrightarrow{\ell^{\prime}}\right)\right\rangle=(2 \pi)^{2} \mathcal{C}^{\phi \phi}\left(k, k^{\prime}, \ell\right) \delta_{D}^{2}\left(\vec{\ell}-\vec{\ell}^{\prime}\right)$ and $\left\langle\phi_{\ell m}(k) \phi_{\ell^{\prime} m^{\prime}}^{*}\left(k^{\prime}\right)\right\rangle=C_{\ell}^{\phi \phi}\left(k, k^{\prime}\right) \delta_{\ell \ell^{\prime}} \delta_{m m^{\prime}}$.

One then has the following identical expressions for the flat-sky power spectra of the shear and the convergence fields 42 ]

$$
\begin{aligned}
& \mathcal{C}^{\gamma \gamma}\left(k_{1}, k_{2}, \ell\right)=\frac{\ell^{4}}{4} \mathcal{C}^{\phi \phi}\left(k_{1}, k_{2}, \ell\right), \\
& \mathcal{C}^{\kappa \kappa}\left(k_{1}, k_{2}, \ell\right)=\frac{\ell^{4}}{4} \mathcal{C}^{\phi \phi}\left(k_{1}, k_{2}, \ell\right) .
\end{aligned}
$$

where now

$$
\mathcal{C}^{\phi \phi}\left(k_{1}, k_{2}, \ell\right)=\frac{16}{\pi^{2} c^{4}} \int_{0}^{\infty} k^{2} d k I\left(k_{1}, k\right) I\left(k_{2}, k\right)
$$

with $I$ as before (see Eq. (53)). The equations (64), (66) and (53) open up the possibility of cosmological parameter estimation with the shear field, whose 2-point statistics are here related to those of the underlying gravitational potential field (and hence to the matter density via Poisson's equation). For clarity, we have not considered here the practical issues of noise, source selection function, weighting schemes, or errors in the photometric distance indicators. These can be dealt with, and are studied in a companion paper [69], which addresses the accuracy with which cosmological parameters can be estimated with current and future 3D weak lensing surveys. Also considered in that paper is that one has to assume a fiducial cosmological model in order to translate source redshifts into comoving distance coordinates $r$.

In Figures (11) to (4) we illustrate the amplitude and shape of the signal produced by the 3D shear power spectrum $\mathcal{C}^{\gamma \gamma}\left(k_{1}, k_{2}, \ell\right)$ of Eq. (64) one may ideally expect to measure. We also show how the shear power spectrum varies with the value of the equation of state parameter $w$. The plots are for a fiducial $\Lambda$ CDM cosmological model with parameters $\Omega_{\Lambda}=0.73, \Omega_{m}=0.27, \Omega_{b}=0.27, H_{0}=71 \mathrm{~km} / \mathrm{s} / \mathrm{Mpc}$ and equation of state of dark energy parameter $w=-1$. Figs. (11), (2) and (3) show the features of the 3D shear power spectrum by taking various cuts through the $3 \mathrm{D}\left(k_{1}, k_{2}, \ell\right)$ space. We consider a survey to $r_{\max }=5000 \mathrm{Mpc}$, which replaces infinity as the upper limit of the radial integral in Eq. (53). The sampling interval in wavenumber $k$ is $2.5 \times 10^{-4} \mathrm{Mpc}^{-1}$ and in multipole $\log _{10}(\ell)$ is 0.025 . We also illustrate in Fig. (4) how the shear power spectrum varies with $w$. The differences are slight in an individual power spectrum, but as this is a 3D study, there are many useful $\ell$ modes, which increase the sensitivity of the 3D shear as a cosmological parameter diagnostic.

\section{3D CORRELATION FUNCTION}

For surveys with complicated geometry, the 3D shear correlation function may be a more appropriate tool to use, in place of the 3D shear power spectrum. It is straightforward to relate the 3D shear correlation function to the lensing potential power spectrum (and hence the matter power spectrum), via (52), as follows. The shear expansion as defined in Section

$$
\left\langle\gamma(\boldsymbol{r}) \gamma^{*}\left(\boldsymbol{r}^{\prime}\right)\right\rangle=\sum_{\ell m \ell^{\prime} m^{\prime}} \int d k d k^{\prime}\left\langle{ }_{2} \gamma_{k \ell m} \gamma_{2} \gamma_{k^{\prime} \ell^{\prime} m^{\prime}}^{*}\right\rangle_{2} Z_{k \ell m}(r, \theta, \varphi){ }_{2} Z_{k^{\prime} \ell^{\prime} m^{\prime}}^{*}\left(r^{\prime}, \theta^{\prime}, \varphi^{\prime}\right)
$$

Using [48), this reduces to

$$
\left\langle\gamma(\boldsymbol{r}) \gamma^{*}\left(\boldsymbol{r}^{\prime}\right)\right\rangle=\frac{2}{\pi} \sum_{\ell m} \frac{1}{4} \frac{(\ell+2) !}{(\ell-2) !} \int d k d k^{\prime} C_{\ell}^{\phi \phi}\left(k, k^{\prime}\right) k k^{\prime} j_{\ell}(k r) j_{\ell}\left(k^{\prime} r^{\prime}\right){ }_{2} Y_{\ell m}(\theta, \varphi){ }_{2} Y_{\ell m}^{*}\left(\theta^{\prime}, \varphi^{\prime}\right) .
$$

Using the generalised addition theorem for spin- $s$ spherical harmonics [76], we find

$$
\left\langle\gamma(\boldsymbol{r}) \gamma^{*}\left(\boldsymbol{r}^{\prime}\right)\right\rangle=\frac{1}{2 \pi} \sum_{\ell} \sqrt{\frac{2 \ell+1}{4 \pi}} \frac{(\ell+2) !}{(\ell-2) !} \int d k d k^{\prime} C_{\ell}^{\phi \phi}\left(k, k^{\prime}\right) k k^{\prime} j_{\ell}(k r) j_{\ell}\left(k^{\prime} r^{\prime}\right)_{2} Y_{\ell,-2}(\beta, \alpha) e^{-2 i \epsilon},
$$

where $\beta$ is the angle between the directions $(\theta, \varphi)$ and $\left(\theta^{\prime}, \varphi^{\prime}\right)$, and $\alpha$ and $\epsilon$ are the angles between the line joining the two points on the celestial sphere and the lines of constant $\varphi$. An explanatory diagram can be found in [76]. Note that one might expect that the correlation function would depend only on the angular separation through $\beta$, but as $\gamma$ 
$\ell=20$

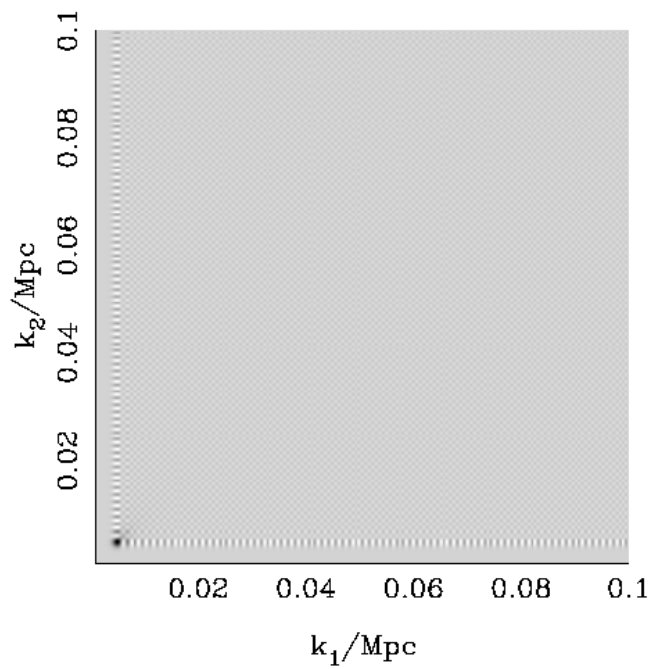

$\ell=20 \quad \mathrm{k}_{1}=0.02$

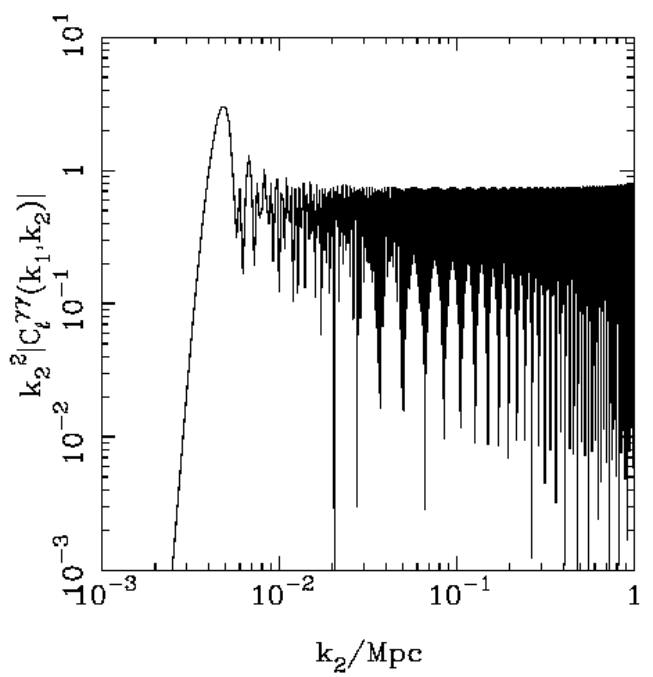

FIG. 1: The left-hand panel shows a grayscale cross-section through the $\mathcal{C}^{\gamma \gamma}\left(k_{1}, k_{2}, \ell\right)$ 3D matrix where each element has been multiplied by $k_{1} k_{2}$. The black/white colour represents positive/negative values. The right-hand panel represents a cut in the $\left(k_{1}, k_{2}\right)$ plane for a given value of $\ell$ and $k_{1}$. The fiducial cosmological model parameters chosen are $\Omega_{\Lambda}=0.73, \Omega_{m}=0.27$, $\Omega_{b}=0.27, H_{0}=71 \mathrm{~km} / \mathrm{s} / \mathrm{Mpc}$ and $w=-1$.

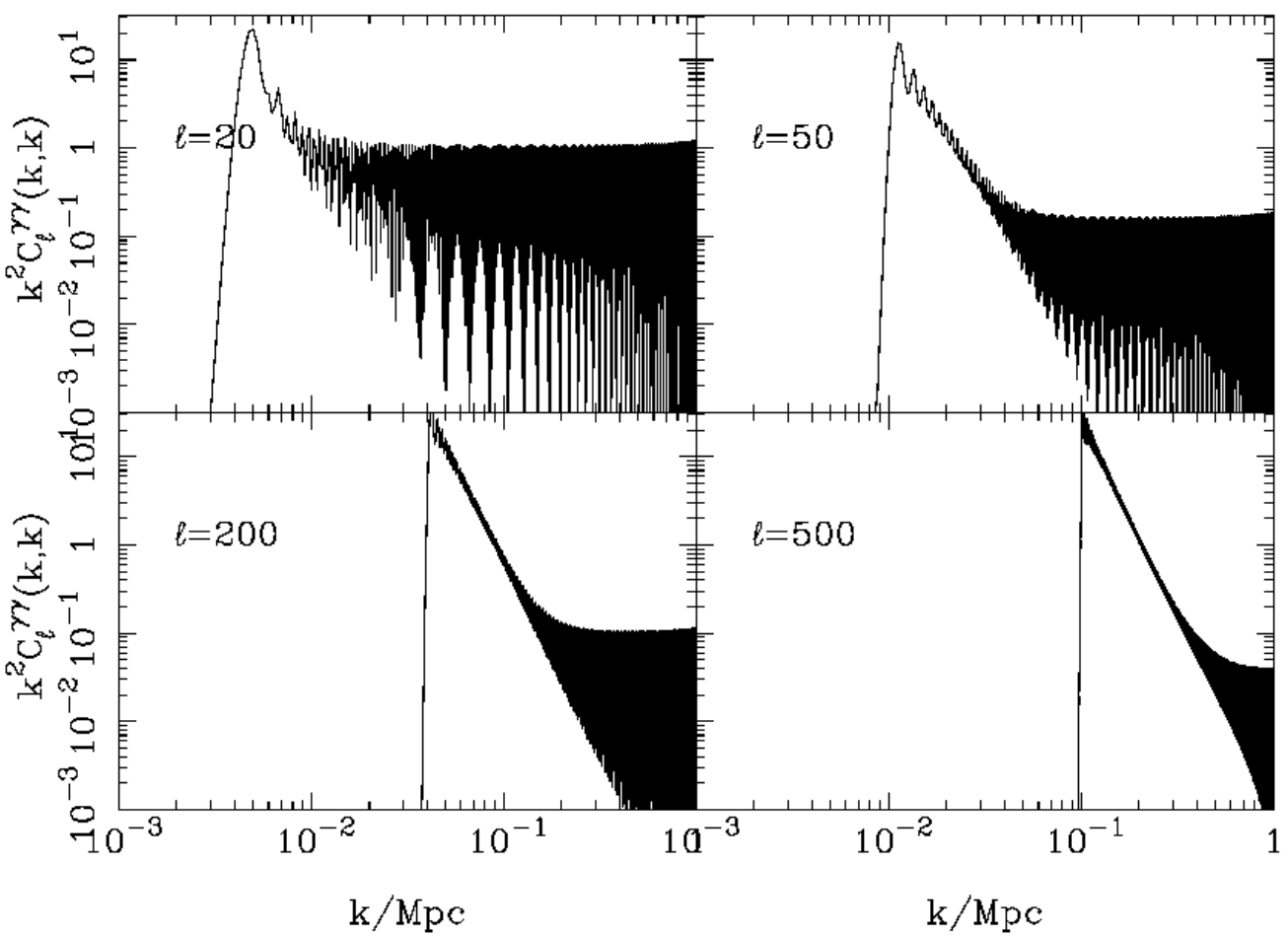

FIG. 2: These plots show diagonal cuts in the $\left(k_{1}, k_{2}\right)$ plane at different $\ell$ values through the $\mathcal{C}^{\gamma \gamma}\left(k_{1}, k_{2}, \ell\right) 3 \mathrm{D}$ matrix. The effect of the approximate Bessel function inequality, $k r \geq \ell$, in Eq. (53) can be seen. As the $\ell$ value increases the diagonal terms of the covariance do not become significant until $k r_{\max } \approx \ell$, where $r_{\max }$ is the upper limit imposed on the $r$ integral. The fiducial cosmological model chosen is the same as in Fig. (1). 


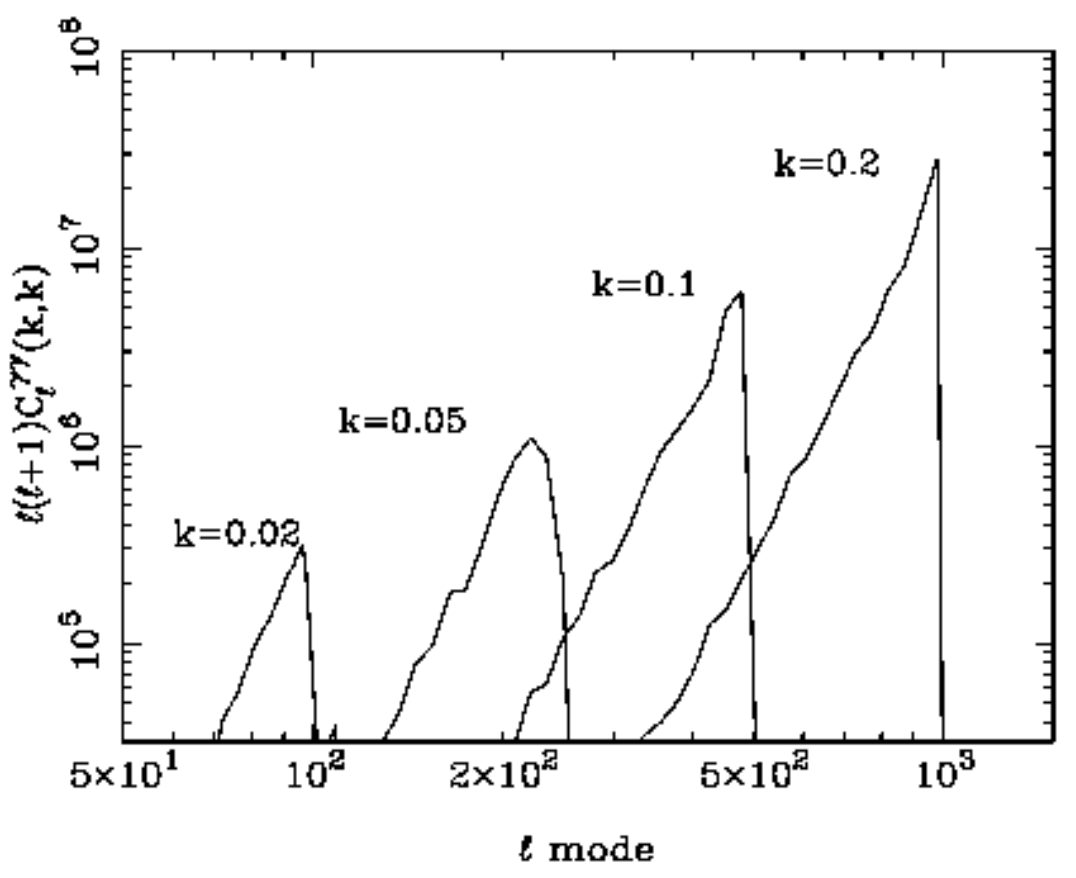

FIG. 3: Dependence of the diagonal part of the 3D shear power spectrum $\mathcal{C}^{\gamma \gamma}\left(k_{1}, k_{2}, \ell\right)$ on $\ell$, for various $k$ values in Mpc ${ }^{-1}$. The fiducial cosmological model chosen is the same as in Fig. (1).

is defined with reference to the orthogonal coordinate system $(\theta, \varphi)$, there is an additional dependence on the angles $\alpha$ and $\epsilon$. Note also that the 3D correlation function is not homogeneous. If one chooses, one may simplify slightly by considering pair correlations of the shear defined with respect to axes parallel and perpendicular to a great circle joining the two galaxies on the sky. The rotation of the $\gamma$ coefficients (to $\tilde{\gamma}$ ) then gives

$$
\left\langle\tilde{\gamma}(\boldsymbol{r}) \tilde{\gamma}^{*}\left(\boldsymbol{r}^{\prime}\right)\right\rangle=\frac{1}{2 \pi} \sum_{\ell} \sqrt{\frac{2 \ell+1}{4 \pi}} \frac{(\ell+2) !}{(\ell-2) !} \int d k d k^{\prime} C_{\ell}^{\phi \phi}\left(k, k^{\prime}\right) k k^{\prime} j_{\ell}(k r) j_{\ell}\left(k^{\prime} r^{\prime}\right){ }_{2} Y_{\ell,-2}(\beta, 0)
$$

where ${ }_{2} Y_{\ell,-2}(\theta, 0)$ may be simplified to a weighted sum of 3 associated Legendre functions.

One can derive equivalent flat-sky relations by using the expressions (14) for the weak-lensing shear on the flat-sky and the expansion (57) for the lensing potential. Using the result $\gamma(k, \vec{\ell})=\left[\left(\ell_{x}^{2}-\ell_{y}^{2}\right) / 2+i \ell_{x} \ell_{y}\right] \phi(k, \vec{\ell})$, where $\vec{\ell}$ and $k$ are defined as in the previous section, we easily obtain

$$
\left\langle\gamma(r, \vec{\theta}) \gamma^{*}\left(r^{\prime}, \vec{\theta}^{\prime}\right)\right\rangle=\frac{1}{8 \pi^{3}} \int d^{2} \vec{\ell} \ell^{4} \int d k d k^{\prime} \mathcal{C}^{\phi \phi}\left(k, k^{\prime}, \ell\right) k k^{\prime} j_{\ell}(k r) j_{\ell}\left(k^{\prime} r^{\prime}\right) e^{i \vec{\ell} \cdot\left(\vec{\theta}-\vec{\theta}^{\prime}\right)}
$$

where $\mathcal{C}^{\phi \phi}\left(k, k^{\prime}, \ell\right)$ is the flat-sky power spectra of the lensing potential. Alternatively, one can reach the same result by expanding Eq. (69) around the pole of spherical coordinates $\theta \simeq 0$ and taking the continuous limit of high multipoles $\ell$.

\section{CONCLUSIONS}

In this paper we have developed in detail a complete formal study of $3 \mathrm{D}$ weak lensing, where one has estimates of the weak lensing shear field at known locations in 3D space. Most cosmological weak lensing surveys to date have been analysed in projection on the 2D sky, where the individual distances of the lensed galaxies are ignored. With distance information, a more sophisticated analysis is possible, which offers the prospect of greater statistical power. A partly 3D approach is to divide the sources into redshift slices, a process often referred to as tomography, but this crude division fails to explore the complete potential provided by distance information, and it makes sense to exploit as fully as possible the 3D information available: one essentially has a noisy estimate of the 3D shear field at the positions of all the source galaxies. For the estimation of some parameters, such as the amplitude of the power spectrum, 3D information adds relatively little, but for others, such as the equation of state of dark energy, 3D weak 


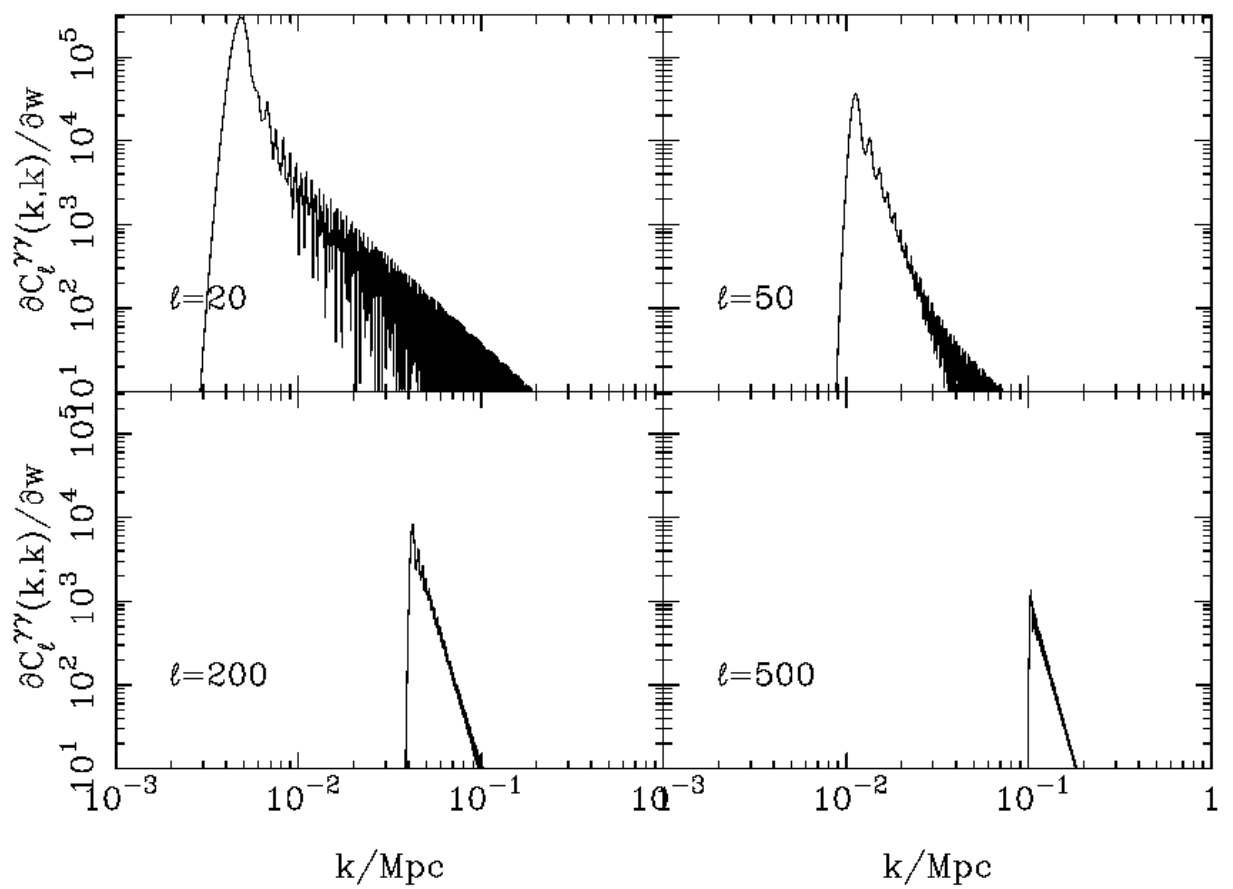

FIG. 4: The derivative of the diagonal elements of the $3 \mathrm{D}$ covariance matrix $\mathcal{C}^{\gamma \gamma}\left(k_{1}, k_{2}, \ell\right)$ for various $\ell$ values, with respect to the dark energy equation of state parameter $w$. The fiducial cosmological model chosen is the same as in Fig. (1) where $w$ is allowed to vary.

lensing analysis is very promising, and this facet of 3D lensing is explored in a companion paper 69. Indeed, weak lensing on a cosmic scale may be the best cosmological method able to answer the important question of the nature of the dark energy.

To effect a 3D analysis, we have used the theory of spin-weight functions successfully applied in the past to studies of the 2D CMB polarisation on the sky 41, 42, 43, 44, 45], as well as theoretical 2D weak-lensing analysis 25, 26, 27]. We have shown that the natural expansion basis to use for the 3D shear field is the product of spin-weight 2 spherical harmonics and spherical Bessel functions, which form a complete and orthonormal system of tensor functions on the three-dimensional space. Alternative expansions are clearly possible such as using tensor spherical harmonics [27] but these are more difficult to work with and have been in some way abandoned by the CMB polarisation community. With our basis choice, the two-point statistics (3D shear power spectrum, 3D correlation function) of the weak lensing 3D shear field can be related in a straightforward manner to the 3D power spectrum of matter density fluctuations. The statistics of the 3D shear field are then connected to cosmological parameters via this relation, in addition to the redshift-distance dependence which enters the transform. This connection is easily shown using the edth differential operators. We are also able to relate the power spectrum of a full-sky 3D shear expansion to the power spectrum of a small-angle survey, where the use of spin-weight spherical harmonics is cumbersome, and a more familiar transverse Fourier expansion can be employed. This is the basis for cosmological parameter estimation using 3D weak lensing, and the formalism presented here provides a useful theoretical framework for 3D weak lensing studies.

\section{Acknowledgments}

PGC is supported by the PPARC through a Postdoctoral rolling grant. TDK acknowledges a PPARC scholarship. 


\section{APPENDIX A: SPIN-WEIGHT $s$ FUNCTIONS AND SPIN RAISING AND LOWERING OPERATORS}

In this Appendix we review the notation and the mathematics of spin-weight $s$ functions and of the geometrical

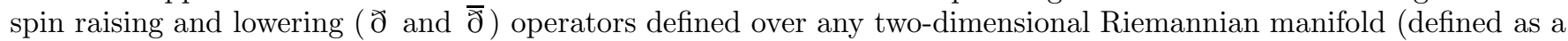
$2 \mathrm{D}$ space with a metric). The relation between the spin-weight functions and tensor quantities is explained. We also clarify the relations between the $\widetilde{\partial}(\bar{\delta})$ operators and covariant derivatives on the 2-dimensional manifold. We then particularise to both the 2-dimensional unit sphere and unit Cartesian space, which will be of use to us in the main body of this work. We finally give an overview of the spherical spin-weight spherical harmonics. Many articles and appendices in the literature have been dedicated to this topic (see [41, 43, 45, 46, 56, 72] to cite just a useful few).

The spin-weight functions and the so-called $e d t h$ ( $\widetilde{\partial}$ and $\bar{\varnothing}$ ) operators were first introduced in the 60 s by Newman \& Penrose [46] and further explored by Goldberg et al. 56]. They mainly started to be used as a convenient tool in the context of the theory of gravitational wave radiation (see e.g. K.S. Thorne 73]) and later they were introduced in the study of the Cosmic Microwave Background (CMB) all-sky polarisation as an alternative to the tensor formalism (see [1] ). The similarity between the CMB polarisation Stokes parameters $Q$ and $U$ and the weak lensing components $\gamma_{1}$ and $\gamma_{2}$ as well as the perspective of future large-field high resolution observations triggered recent full-sky weak lensing studies using the spin-weight formalism [24, 42, 53].

\section{Representation of spin-weight functions in $2 \mathrm{D}$ Riemannian manifolds}

Let us define an orthonormal basis at any point of a two dimensional manifold with a metric $g_{i j}\left\{\hat{\boldsymbol{e}}_{\mathbf{1}}, \hat{\boldsymbol{e}}_{\mathbf{2}}\right\}$. The choice of the basis is not unique. Indeed, if we define the vectors $\boldsymbol{m}$ and $\overline{\boldsymbol{m}}$ with respect to $\left\{\hat{\boldsymbol{e}}_{\mathbf{1}}, \hat{\boldsymbol{e}}_{\mathbf{2}}\right\}$, the choice of the basis is described up to a phase $\psi$ such that

$$
\begin{aligned}
& \boldsymbol{m}=\frac{1}{\sqrt{2}}\left(\hat{\boldsymbol{e}}_{\mathbf{1}}+i \hat{\boldsymbol{e}}_{\mathbf{2}}\right) \rightarrow e^{-i \psi} \boldsymbol{m}, \\
& \overline{\boldsymbol{m}}=\frac{1}{\sqrt{2}}\left(\hat{\boldsymbol{e}}_{\mathbf{1}}-i \hat{\boldsymbol{e}}_{\mathbf{2}}\right) \rightarrow e^{i \psi} \overline{\boldsymbol{m}} .
\end{aligned}
$$

The vector $\boldsymbol{m}$ and its complex conjugate $\overline{\boldsymbol{m}}$ obey the relations $\boldsymbol{m}^{j} \boldsymbol{m}_{j}=\overline{\boldsymbol{m}}^{j} \overline{\boldsymbol{m}}_{j}=0, \boldsymbol{m}^{j} \overline{\boldsymbol{m}}_{j}=1$ and $\boldsymbol{m}^{i} \overline{\boldsymbol{m}}_{j}+$ $\overline{\boldsymbol{m}}^{i} \boldsymbol{m}_{j}=\delta_{j}^{i}$, where $\delta_{j}^{i}$ is the Kronecker delta function. Here and throughout this manuscript, we use the Einstein summation convention.

A complex function defined by ${ }_{s} f(\hat{\boldsymbol{n}}) \equiv f_{1}(\hat{\boldsymbol{n}})+i f_{2}(\hat{\boldsymbol{n}})$, where $f_{1}$ and $f_{2}$ are real quantities defined on the manifold, is said to have spin-weight $s$ if under the transformation Eq. (A1) it transforms as ${ }_{s} f(\hat{\boldsymbol{n}}) \rightarrow e^{-i s \psi}{ }_{s} f(\hat{\boldsymbol{n}})$. If we consider an arbitrary vector on the manifold $\boldsymbol{v}(\hat{\boldsymbol{n}})$ then, for instance, the quantities $\boldsymbol{v} . \hat{\boldsymbol{e}}_{\mathbf{1}} \pm i \boldsymbol{v} . \hat{\boldsymbol{e}}_{\mathbf{2}}$ transform as spinweight \pm 1 quantities. Generalising to a rank-s tensor $F_{i_{1} \ldots i_{s}}$ (where $i=0,1$ ), the object $F_{i_{1} \ldots i_{s}} \boldsymbol{m}^{i_{1}} \ldots \boldsymbol{m}^{i_{s}}$ transforms as a spin-weight $s$ object as each individual vector $\boldsymbol{m}^{i_{n}}$ contributes with a factor $e^{-i \psi}$ when it is transformed. The rank of the tensor is thus reflected in the transformation properties, and thus the spin, of the corresponding complex quantity constructed.

One can therefore associate to every symmetric and trace-free component of a rank $s$ tensor a spin-weight $s$ quantity such that

$$
\begin{aligned}
{ }_{s} f(\hat{\boldsymbol{n}}) & =F_{i_{1} \ldots i_{s}} \boldsymbol{m}^{i_{1}} \ldots \boldsymbol{m}^{i_{s}} \\
{ }_{-s} f(\hat{\boldsymbol{n}}) & =F_{i_{1} \ldots i_{s}} \overline{\boldsymbol{m}}^{i_{1}} \ldots \overline{\boldsymbol{m}}^{i_{s}} .
\end{aligned}
$$

The trace-free condition refers to the vanishing under contraction of any two indices in the tensor. For example, for a rank-2 tensor, it refers to $F_{i}^{i}=F_{i i^{\prime}} g^{i^{\prime} i}$. As irreducible tensors of rank $s$ in 2 dimensions have only two linearly independent components, say $F_{00 \ldots 0}$ and $F_{10 \ldots 0}$, we can combine them to create the associated spin-weight $\pm s$ complex quantities $\pm s f(\hat{\boldsymbol{n}})=F_{00 \ldots 0} \pm i F_{10 \ldots 0}$. It is a one-to-one mapping. Conversely, one can express any symmetric and trace-free rank $s$ tensor in terms of spin-weight $\pm s$ objects

$$
F_{i_{1} \ldots i_{s}}={ }_{s} f(\hat{\boldsymbol{n}}) \overline{\boldsymbol{m}}_{i_{1}} \ldots \overline{\boldsymbol{m}}_{i_{s}}+{ }_{-s} f(\hat{\boldsymbol{n}}) \boldsymbol{m}_{i_{1} \ldots \boldsymbol{m}_{i_{s}}}
$$

An important property of spin-weight objects is that there exists a geometrical spin-raising (lowering) operator $\delta$ $(\overline{\widetilde{\delta}})$ that have the ability of raising (lowering) the spin-weight $s$ of an object such that under the transformation (A1)

$$
\begin{aligned}
& \partial\left[{ }_{s} f(\hat{\boldsymbol{n}})\right] \rightarrow e^{-i(s+1) \psi} \partial\left[{ }_{s} f(\hat{\boldsymbol{n}})\right], \\
& \overline{\mathrm{\partial}}\left[{ }_{s} f(\hat{\boldsymbol{n}})\right] \rightarrow e^{-i(s-1) \psi \overline{\mathrm{\partial}}}\left[{ }_{s} f(\hat{\boldsymbol{n}})\right] \text {. }
\end{aligned}
$$


Most relevant is that these spin-raising and lowering operators are related to the covariant derivatives of the rank- $s$ tensor associated to the spin-weight $s$ object on the manifold

$$
\begin{aligned}
& \left.\check{\partial}{ }_{s} f(\hat{\boldsymbol{n}})\right]=-\sqrt{2} \boldsymbol{m}^{i_{1}} \ldots \boldsymbol{m}^{i_{s}} \boldsymbol{m}^{k} \nabla_{k} F_{i_{1} \ldots i_{s}}, \\
& \bar{\partial}\left[{ }_{s} f(\hat{\boldsymbol{n}})\right]=-\sqrt{2} \boldsymbol{m}^{i_{1}} \ldots \boldsymbol{m}^{i_{s}} \overline{\boldsymbol{m}}^{k} \nabla_{k} F_{i_{1} \ldots i_{s}}
\end{aligned}
$$

for $s \geq 0$, and with $\boldsymbol{m}^{i_{n}}$ replaced by $\overline{\boldsymbol{m}}^{i_{n}}$ for $s<0$. The choice of the sign and the normalisation are conventional but were chosen so that the form of the spin-raising and lowering operators on the $2 \mathrm{D}$ sphere match the original definition of Newman \& Penrose [46] (see next section). The covariant derivative is defined as usual. For a rank-1 tensor $X_{i}$, for example, $\nabla_{j} X_{i}=\partial_{j} X_{i}-\Gamma_{j i}^{k} X_{k}$ with the Christoffel symbol depending on the metric. If one replaces Eq. (A3) into Eq. A5, one can rewrite Eq. (A5) for $s \geq 0$ as

$$
\begin{aligned}
& \partial\left[{ }_{s} f(\hat{\boldsymbol{n}})\right]=-\sqrt{2}\left(\nabla_{k}\left[{ }_{s} f(\hat{\boldsymbol{n}})\right] \boldsymbol{m}^{k}+s \tau_{s} f(\hat{\boldsymbol{n}})\right), \\
& \bar{\partial}\left[{ }_{s} f(\hat{\boldsymbol{n}})\right]=-\sqrt{2}\left(\nabla_{k}\left[{ }_{s} f(\hat{\boldsymbol{n}})\right] \overline{\boldsymbol{m}}^{k}-s \bar{\tau}_{s} f(\hat{\boldsymbol{n}})\right)
\end{aligned}
$$

where

$$
\tau=\nabla_{k} \overline{\boldsymbol{m}}_{i} \boldsymbol{m}^{i} \boldsymbol{m}^{k}
$$

The equivalent relations for $s<0$ follow from these. One can express any differential operator in two dimensions in terms of $\check{\partial}$ and $\bar{\partial}$. For instance for a spin-weight s field ${ }_{s} f$, we have the following relation between the $\check{\partial}$ operators and the Laplacian operator

$$
\nabla^{2}\left[{ }_{s} f\right]=\frac{1}{2}[ð \bar{\partial}+\bar{\partial} \partial]_{s} f .
$$

\section{Representation of spin-weight functions on the unit sphere}

We choose the orthonormal basis on the sphere to be aligned with the coordinate basis vectors $\hat{\boldsymbol{\theta}}$ and $\hat{\boldsymbol{\varphi}}$ of a spherical polar coordinate system $\left\{\hat{\boldsymbol{e}}_{\boldsymbol{\theta}}(\hat{\boldsymbol{n}}), \hat{\boldsymbol{e}}_{\boldsymbol{\varphi}}(\hat{\boldsymbol{n}})\right\}$ where $\hat{\boldsymbol{n}}$ is the radial direction vector normal to the surface of the sphere. The transformation defined in Eq. (A1) corresponds to a right-handed rotation by an angle $\psi$ of this basis around the vector $\hat{\boldsymbol{n}}$. The convention chosen for the rotation is the same as in Zaldarriaga \& Seljak [41] and Okamoto \& $\mathrm{Hu}$ [45] but differs from Newman \& Penrose [46] and Goldberg et al. [56] so that it conforms to the majority of CMB polarisation publications.

The metric tensor is given by

$$
g_{i j}=\left(\begin{array}{cc}
1 & 0 \\
0 & \sin ^{2} \theta
\end{array}\right)
$$

and the nonzero Christoffel symbols are

$$
\Gamma_{11}^{0}=-\sin \theta \cos \theta, \Gamma_{01}^{1}=\Gamma_{10}^{1}=\cot \theta .
$$

The tensor components of the vector $\boldsymbol{m}$ (see Eq. (A1)) are chosen to be

$$
\boldsymbol{m}^{k}=\frac{1}{\sqrt{2}}(1, i \csc \theta), \quad \boldsymbol{m}_{k}=\frac{1}{\sqrt{2}}(1, i \sin \theta) .
$$

To obtain the exact relation for the spin-raising and lowering operators $\bar{\partial}$ and $\bar{\delta}$ on the sphere we use Eqs. (A6) and (A7). To calculate $\tau$ we need the evaluate explicitly the covariant derivatives $\nabla_{i} \overline{\boldsymbol{m}}_{j}$ on the spherical basis with coordinates $(\hat{\boldsymbol{\theta}}, \hat{\boldsymbol{\varphi}})$. We obtain

$$
\begin{aligned}
\nabla_{\theta} \overline{\boldsymbol{m}}_{\varphi} & =0 \\
\nabla_{\varphi} \overline{\boldsymbol{m}}_{\theta} & =\frac{i}{\sqrt{2}} \cos \theta \\
\nabla_{\varphi} \overline{\boldsymbol{m}}_{\varphi} & =\frac{1}{\sqrt{2}} \sin \theta \cos \theta
\end{aligned}
$$


such that

$$
\tau=\frac{-\cot \theta}{\sqrt{2}}
$$

yielding Eqs. (18) and (19). We note that the expressions obtained are identical to expressions found elsewhere 41, 43, 45, 46]. This stems from the choice of a suitable formalism convention. Another useful result is the full expression of the Laplacian acting on a spin-0 quantity $\phi$

$$
\nabla^{2} \phi=\frac{1}{2}[ð \bar{\partial}+\bar{\partial} \partial] \phi=\left[\nabla_{\theta} \nabla_{\theta}+\csc ^{2} \theta \nabla_{\varphi} \nabla_{\varphi}\right] \phi .
$$

We remark that $\nabla^{2}$ corresponds to $-\ell(\ell+1)$ in spherical harmonic space.

\section{Representation of spin-weight functions in Euclidean space}

We will also be concerned with fields defined over a 2-dimensional Euclidean space in the so-called flat-sky approximation for which the natural basis is the Cartesian coordinate system $\left\{\hat{\boldsymbol{e}}_{\boldsymbol{x}}(\hat{\boldsymbol{n}}), \hat{\boldsymbol{e}}_{\boldsymbol{y}}(\hat{\boldsymbol{n}})\right\}$ where $\hat{\boldsymbol{n}}$ is the vector normal to the surface of the sky. In this case, the metric tensor is given by

$$
g_{i j}=\left(\begin{array}{ll}
1 & 0 \\
0 & 1
\end{array}\right) .
$$

The tensor components of the vector $\boldsymbol{m}$ (see Eq. A1) are chosen to be

$$
\boldsymbol{m}^{k}=\frac{1}{\sqrt{2}}(1, i)
$$

where $\boldsymbol{m}^{k}=\boldsymbol{m}_{k}$. The Christoffel symbols are all zero and thus in Eqs. A6 and A7 we have that $\tau=0$. In this case, the differential operators $\partial$ and $\bar{\partial}$ naturally reduce to Eqs. [201 [26]. For completeness, the Laplacian in Cartesian coordinates reduces to

$$
\nabla^{2} \phi=\frac{1}{2}[ð \bar{\partial}+\bar{\partial} \check{\partial}] \phi=\left[\partial_{x}^{2}+\partial_{y}^{2}\right] \phi .
$$

\section{APPENDIX B: SPIN WEIGHT SPHERICAL HARMONICS}

A scalar field defined on the sphere can be expanded in spherical harmonics $Y_{\ell m}(\theta, \varphi)$, which form a complete and orthonormal basis on the sphere. This basis of spherical harmonics is no longer appropriate to describe objects of spin-weight $s$. There exists similar sets of functions defined on the sphere that can be used instead. These are called spin-weight spherical harmonics ${ }_{s} Y_{\ell m}(\theta, \varphi)$ and are defined by [74]

$$
{ }_{s} Y_{\ell m}(\theta, \varphi)=\sqrt{\frac{(2 \ell+1)}{4 \pi}} D_{-s, m}^{\ell}(\theta, \varphi, 0)
$$

where $D$ is the Wigner-D function (for technical details see [57, 75]). Using the previous relation one can prove that the spin-weight $s$ spherical harmonics satisfy the relation of orthogonality

$$
\int_{0}^{2 \pi} d \varphi \int_{-1}^{1} d \cos \theta_{s^{\prime}} Y_{\ell^{\prime} m^{\prime}}^{*}(\theta, \varphi){ }_{s} Y_{\ell m}(\theta, \varphi)=\delta_{\ell \ell^{\prime}} \delta_{m m^{\prime}} \delta_{s s^{\prime}}
$$

where $\delta_{i j}$ is a Kronecker delta function. Also ${ }_{s} Y_{\ell m}^{*}=(-1)^{s}{ }_{-s} Y_{\ell-m}$. A given spin-weight $s$ function on the $3 \mathrm{D}$ sphere can thus be expanded as [49]

$$
\begin{aligned}
{ }_{s} f(\boldsymbol{x}) & =\int_{0}^{\infty} d k \sum_{\ell=0}^{\infty} \sum_{m=-\ell}^{\ell}\left[a_{s, \ell m}(k)\right]_{s} Z_{k \ell m}(x, \theta, \varphi), \\
a_{s, \ell m}(k) & =\int d^{3} x\left[{ }_{s} f(\boldsymbol{x})\right]_{s} Z_{k \ell m}^{*}(x, \theta, \varphi) .
\end{aligned}
$$


where in spatially flat geometry the basis functions ${ }_{s} Z_{k \ell m}$ are given by combinations of spherical Bessel functions and spin-weight $s$ spherical harmonics as in Eq. (33). They are orthonormal

$$
\int d^{3} x_{s} Z_{k \ell m}(x, \theta, \varphi)_{s^{\prime}} Z_{k^{\prime} \ell^{\prime} m^{\prime}}^{*}(x, \theta, \varphi)=\delta_{D}\left(k-k^{\prime}\right) \delta_{\ell \ell^{\prime}} \delta_{m m^{\prime}} \delta_{s s^{\prime}}
$$

where we have used Eq. (B2) and the relation

$$
\int x^{2} d x\left[\sqrt{\frac{2}{\pi}} k j_{\ell}(k x)\right]\left[\sqrt{\frac{2}{\pi}} k^{\prime} j_{\ell}\left(k^{\prime} x\right)\right]=\delta_{D}\left(k-k^{\prime}\right) .
$$

If we are not assuming a spatially flat space then the radial functions need to be changed. For an open Universe for example $j_{\ell}(x) \rightarrow X_{\ell}\left(\Omega_{K}, x\right)$ where $X_{\ell}$ is the hyper-spherical Bessel function (see Liddle \& Lyth [49] for details).

A few properties of spin-weighted spherical harmonics when acted by spin-lowering and rising operators (as defined in Eqs. (18) and (19)) can be very useful

$$
\begin{aligned}
& \mathrm{\partial}_{s} Y_{\ell m}=[(\ell-s)(\ell+s+1)]^{\frac{1}{2}}{ }_{s+1} Y_{\ell m}, \\
& \overline{\widetilde{\partial}}_{s} Y_{\ell m}=-[(\ell+s)(\ell-s+1)]^{\frac{1}{2}}{ }_{s-1} Y_{\ell m} \text {, } \\
& \bar{\partial} \partial_{s} Y_{\ell m}=-(\ell-s)(\ell+s+1){ }_{s} Y_{\ell m} \text {, } \\
& \text { ठ } \bar{\partial}{ }_{s} Y_{\ell m}=-(\ell+s)(\ell-s+1){ }_{s} Y_{\ell m} \text {. }
\end{aligned}
$$

In particular, the spin-0 and spin-2 spherical harmonics are related through

$$
\begin{aligned}
& \text { ठठ } Y_{\ell m}=\sqrt{\frac{(\ell+2) !}{(\ell-2) !}}{ }_{2} Y_{\ell m}, \\
& \bar{\varnothing} \bar{\partial} Y_{\ell m}=\sqrt{\frac{(\ell+2) !}{(\ell-2) !}}-{ }_{2} Y_{\ell m} .
\end{aligned}
$$

A useful consequence of these properties is

$$
\bar{\partial} \bar{\partial} \check{\partial} Y_{\ell m}=ð ð \bar{\partial} \bar{\partial} Y_{\ell m}=\frac{(\ell+2) !}{(\ell-2) !} Y_{\ell m}=(\ell+2)(\ell+1) \ell(\ell-1) Y_{\ell m}
$$

which reduces to (see e.g. [44])

$$
\bar{ð} \bar{\partial} \succsim=ð ð \bar{\partial} \bar{\partial}=\nabla^{2}\left(\nabla^{2}+2\right)
$$

when acting on spin-zero variables and where $\nabla$ is a covariant derivative on the sphere.

[1] M. Bartelmann and P. Schneider, Phys. Rep. 340, 291 (2001)

[2] L. Van Waerbeke and Y. Mellier, in Gavitational lensing: a unique tool for cosmology, Proceedings of the Aussois winter school, edited by D. Walls-Gabaud and J.-P. Kneib (ASP Conference Series, 2003)

[3] D. Bacon, A. Refregier, and R. Ellis, Mon. Not. Roy. Astron. Soc. 318, 625 (2000)

[4] L. van Waerbeke et al., Astron. Astrophys. 374, 757 (2001)

[5] H. Hoekstra et al., Astrophys. J. 572, 55 (2002)

[6] T. Hamana et al., Astrophys. J. 597, 98 (2003)

[7] M. Brown et al., Mon. Not. Roy. Astron. Soc. 341, 100 (2003)

[8] M. Jarvis et al., Astrophys. J. 125, 1014 (2003)

[9] W. Hu and S. Dodelson, Ann. Rev. Astron. Astrophys. 40, 171 (2002)

[10] D. Spergel et al., Astrophys. J. Supp. 148, 175 (2003)

[11] L. Verde et al., Mon. Not. Roy. Astron. Soc. 335, 432 (2002)

[12] S. Cole et al. (submitted to Mon. Not. Roy. Astron. Soc. , 2005)

[13] D.J. Eisenstein et al. (submitted to Astrophys. J. , 2004)

[14] G. Aldering et al. (submitted to PASP, 2004)

[15] Perlmutter, S. et al., Astrophys. J. 517, 565 (1999)

[16] See the webpage: http://dls.bell-labs.com/ 
[17] See the webpage: http://www.noao.edu/noao/noaodeep/

[18] See the webpage: http://www.cfht.hawaii.edu/Science/CFHLS/

[19] See the webpage: http://snap.lbl.gov/

[20] See the webpage: http://pan-starrs.ifa.hawaii.edu/

[21] See the webpage: http://www.lsst.org/lsst_home.shtml

[22] M. Jarvis, B. Jain, G. Bernstein, and D. Dolney (submitted, 2005)

[23] M. Ishak (submitted, 2005)

[24] A. Heavens, Mon. Not. Roy. Astron. Soc. 343, 1327 (2003)

[25] M. Kamionkowski et al., Mon. Not. Roy. Astron. Soc. 301, 1064 (1998)

[26] R.G. Crittenden et al., DAMTP-2000-135 (2000)

[27] A. Stebbins (submitted, 1996)

[28] H.M.P. Couchman et al., Mon. Not. Roy. Astron. Soc. 308, 180 (1999)

[29] A.J. Barber et al., Mon. Not. Roy. Astron. Soc. 319, 267 (2000)

[30] D.J. Bacon (submitted to Mon. Not. Roy. Astron. Soc. , 2004)

[31] D. Wittman et al., Astrophys. J. Lett. 557, L89 (2001)

[32] D. Wittman et al., Astrophys. J. Lett. 597, 218 (2003)

[33] A.N. Taylor et al. (submitted to Mon. Not. Roy. Astron. Soc. , 2003)

[34] W. Hu, Astrophys. J. 522, L21 (1999)

[35] F. Bernardeau, Y. Mellier, and L. van Waerbeke, Astron. Astrophys. 389, L28 (2002)

[36] U.-L. Pen et al., Astrophys. J. 592, 664 (2003)

[37] D.J. Bacon and A.N. Taylor, Mon. Not. Roy. Astron. Soc. 344, 1307 (2003)

[38] A.N. Taylor (submitted to Phys. Rev. Lett. , 2001)

[39] W. Hu and C.R. Keeton, Phys. Rev. D 66, 063506 (2002)

[40] W. Hu, Phys. Rev. D 66, 83515 (2002)

[41] M. Zaldarriaga and U. Seljak, Phys. Rev. D 55, 1830 (1997)

[42] W. Hu, Phys. Rev. D 62, 043007 (2000)

[43] A. Lewis, A. Challinor, and N. Turok, Phys. Rev. D 65, 023505 (2002)

[44] E. Bunn et al., Phys. Rev. D 67, 023501 (2003)

[45] T. Okamoto and W. Hu, Phys. Rev. D 67, 083002 (2003)

[46] E. T. Newman and R. Penrose , J. Math. Phys. Vol.7, number 5, 863 (1966)

[47] R. K. Sachs and A. M. Wolfe, Astrophys. J. 147, 73 (1967)

[48] E. T. Vishniac, Astrophys. J. 322, 597 (1987)

[49] A. R. Liddle and D. H. Lyth, Cosmological Inflation and Large-Scale Structure (Cambridge, 2000)

[50] C.-P. Ma and E. Bertschinger, Astrophys. J. 455, 7 (1995)

[51] A. F. Heavens and A. N. Taylor, Mon. Not. Roy. Astron. Soc. 275, 483 (1995)

[52] F. Bernardeau, L. van Waerbeke, and Y. Mellier, Astron. Astrophys. 322, 1 (1997)

[53] W. Hu, Phys. Rev. D 65, 023003 (2002)

[54] P.J.E. Peebles, Principles of Physical Cosmology (Princeton Series in Physics, 1993)

[55] M. Kamionkowski et al., Phys. Rev. Lett. 78, 2058 (1997)

[56] J. N. Goldberg et al., J. Math. Phys. Vol.8, number 11, 2155 (1967)

[57] D.A. Varshalovich, A.N. Moskalev, and V.K. Khersonskii, Quantum Theory of Angular Momentum (World Scientific, 1988)

[58] A. Challinor, to appear in Data analysis in cosmology (Springer LNP, 2004)

[59] A.H. Norton and R. Bartnik, Geometric Implementation of Spherical Harmonics in Computational Techniques and Applications: CTAC99 (1999)

[60] P. Schneider, L. van Waerbeke, and Y. Mellier, Astron. Astrophys. 389, 729 (2002)

[61] D.M. Goldberg and D.J. Bacon (accepted by Astrophys. J. , 2004)

[62] D.J. Bacon, D.M. Goldberg, B. Rowe, and A.N. Taylor (in preparation)

[63] M. Kamionkowski, A. Kosowsky, and A. Stebbins, Phys. Rev. D 55, 7368 (1997)

[64] N. Kaiser, Astrophys. J. 388, 272 (1992)

[65] N. Kaiser, Astrophys. J. 439, L1 (1995)

[66] E. Hivon et al. (submitted to Astrophys. J. , 2001)

[67] N. Kaiser et al. in Clusters of Galaxies, edited by F. Durrett et al. (Editions Frontiere, Gif-sur-Yvette, 1994) p.269

[68] P. Schneider et al., Mon. Not. Roy. Astron. Soc. 296, 873 (1998)

[69] A. F. Heavens and T. D. Kitching (in preparation)

[70] R. E. Smith, J. A. Peacock, A. Jenkins, S. D. M. White, C. S. Frenk, F. R. Pearce, P. A. Thomas, G. P. Efstathiou, H. M. P. Couchman, Mon. Not. Roy. Astron. Soc. 341, 1311 (2003)

[71] M. Santos et al., Mon. Not. Roy. Astron. Soc. 341, 623 (2003)

[72] M. Eastwood and P. Tod, Math. Proc. Camb. Phil. Soc. 92, 317 (1982)

[73] K. S. Thorne, Rev. Mod. Phys. 52, 299 (1980)

[74] W. Hu and M. White, Astrophys. J. 554, 67 (2001)

[75] R. Penrose and W. Rindler, Spinors and Space-time (Cambridge UP, 1984 and 1986), Vols. I and II.

[76] W. Hu \& M. White, Phys. Rev. D 56, 596, 1997 